This item was submitted to Loughborough's Research Repository by the author.

Items in Figshare are protected by copyright, with all rights reserved, unless otherwise indicated.

\title{
Turbulent closure analysis in heated separated and reattached flow using eddy-resolving data
}

\section{PLEASE CITE THE PUBLISHED VERSION}

https://doi.org/10.1063/1.5141750

\section{PUBLISHER}

AIP Publishing

VERSION

AM (Accepted Manuscript)

\section{PUBLISHER STATEMENT}

This article may be downloaded for personal use only. Any other use requires prior permission of the author and AIP Publishing. This article appeared in Physics of Fluids, 32 (4), 045115 and may be found at https://aip.scitation.org/doi/10.1063/1.5141750.

\section{LICENCE}

\section{All Rights Reserved}

\section{REPOSITORY RECORD}

Ellis, Christopher, and Hao Xia. 2020. "Turbulent Closure Analysis in Heated Separated and Reattached Flow Using Eddy-resolving Data”. Loughborough University. https://hdl.handle.net/2134/12205124.v1. 


\section{Turbulent closure analysis in heated separated and reattached flow using eddy-resolving data}

Christopher D. Ellis ${ }^{\text {a) }}$ and Hao Xia (夏浩)

National Centre for Combustion and Aerothermal Technology,

Department of Aeronautical and Automotive Engineering, Loughborough University, Loughborough, LE11 3GR, United Kingdom

(Dated: 1 April 2020)

In this study, we use Large-Eddy Simulations (LES) to provide a platform to investigate the separated and reattached turbulent flow over a heated blunt plate at $R e_{H}=21600$. Surface Nusselt number and flow field data show good agreement with published experiments. The turbulence anisotropy resolved by the LES shows that through the recirculation region the anisotropy develops towards an axisymmetric contraction state in the near-wall profile. In the redeveloping region, profiles show progression towards the plane-strain state. Turbulent closures, providing simple models of the unknown turbulent correlations that arise from the Reynolds-averaging of the Navier-Stokes equations, are routinely applied to complex flows, often with little known about their suitability. The eddy-resolved flow field is used to describe deficiencies in Reynolds-Averaged Navier-Stokes (RANS) modelling using an LES informed Turbulence Transport (LiTT) a priori analysis. The Explicit Algebraic Reynolds Stress Model (EARSM) showed improved agreement, capturing the elevated turbulent stresses in the recirculation region. Closures describing the turbulent heat flux are compared and the Higher-Order Generalised GDH (HOGGDH) closure is discovered to show good agreement with those resolved by the LES, capturing the correct ratio of streamwise to normal turbulent heat flux across the redeveloping boundary layer. An Explicit Algebraic Scalar Flux Model (EASFM) is examined and shows good predictions of the turbulent heat flux angle but underpredicts the magnitude across the recirculation region. An optimal coefficient for the HOGGDH is described to reproduce the turbulent heat flux magnitude from the LES, showing a range of optimal values across the flow.

a)c.d.ellis@lboro.ac.uk 


\section{INTRODUCTION}

The study of turbulent flow and heat transfer in separated and reattached flow regimes is of practical interest to the engineering community. Combustor liners, heat exchangers and turbine blades are just a few examples of where these flows are present and to understand and predict their heat transfer is vital for product lifetimes and performance. High Reynolds numbers, common within these applications, promote instabilities within the shear layer and the onset of turbulence is close to the separation point. The turbulent shear layer enhances the turbulent mixing through the reattachment and redeveloping regions. The complexities of the flow present challenges to commonly used, efficient and low-cost two-equation Reynolds-Averaged Navier Stokes (RANS) approaches where a desire for improved accuracy is sought for component design. Understanding the RANS closures within these flows allows for improved engineering judgement and may provide a foundation for further closure development.

Separation, reattachment and the resulting heat transfer have been experimentally studied in backwards and forward facing steps, expansion tubes and channels, blunt plates and surface mounted obstructions. Heated blunt plate geometries have been addressed by Ota and colleagues $^{1-5}$. Time-averaged Nusselt number distributions scale with $R e^{2 / 3}$ and peak values exist close to the reattachment location ${ }^{1}$. Temperature and velocity profiles have been presented across a blunt plate showing the rise in non-dimensional temperature through the recirculation bubble and the slow redevelopment of the boundary layer post-reattachment ${ }^{1}$. Ota and Nishiyama ${ }^{2}$ presented a universal correlation of peak Nusselt number to Reynolds number, from a range of separated and reattached flow cases. The nose shape of the blunt plate was investigated by Ota and $\mathrm{Kon}^{3}$, where the reattachment and peak Nusselt number location were shown to move towards the leading edge as the apex angle of the nose increased.

At an unsteady level, hotwire anemometry enabled measurements of turbulent statistical data in these flow regimes. Profiles of turbulent normal and shear stesses had shown elevated values within the turbulent shear layer and remain characteristically larger in the redeveloping flow region when compared to those of a standard turbulent flat plate ${ }^{6}$. Turbulent heat flux was investigated by Ota and $\mathrm{Kon}^{4}$, where correlations with the turbulent shear stress enabled predictions of turbulent Prandtl number, $P r_{t}$. The discussed predictions were scattered, however, a trend-line presented a value of unity close to the wall and with values greater than 4 at the edge of the thermal boundary layer. Pulse Dopler Anemometry (PDA) was used by Djilali and Gartshore ${ }^{7}$ to improve turbulence 
measurements in regions of high turbulence intensity showing large values of root-mean-square (rms) velocity in the recirculation region. Kiya and Sasaki ${ }^{8}$ investigated the blunt plate at $R e_{H}=$ 26000 and found the frequency of vortex shedding at $f=0.6 U_{\infty} / L_{R}$. Further work by Kiya and Sasaki ${ }^{9}$ extended the low-frequency analysis of the separation bubble, presenting the response of reversed flow about the reattachment location to the periodic shedding.

Numerical studies have been used to predict the surface heat transfer in heated blunt plate cases. A two-dimensional simulation at $R e_{H}=1000$ identified a correlation between the large scale vortex shedding and the surface heat transfer, but turbulence quantities were overestimated ${ }^{10}$. A three-dimensional simulation was performed by Tafti and Vanka ${ }^{11}$ which showed the reattachment location at $6.36 \mathrm{H}$ downstream of the leading edge, which overestimated the experimental value by $27 \%$. Direct Numerical Simulations (DNS) were used by Yanaoka et al ${ }^{12}$ where a reattachment length of $4.9 H$, for a $R e=5000$, showed good agreement to experiments. Isosurfaces of the second invariant of velocity gradient showed the instability of the turbulent shear layer, where a two dimensional vortex is shown to followed by the formation of three-dimensional structures downstream ${ }^{12}$. Ratios of streamwise to normal components of the turbulent heat flux vector showed a larger contribution from the streamwise component across the flow and close to the wall. Sugawara et al. ${ }^{13}$ used Large-Eddy Simulations (LES) with a Smagorinski Sub-Grid Scale (SGS) model for heat transfer predictions in a symmetric expansion plane channel at $R e=15000$. Time-averaged results showed a good estimation of the short and long recirculation regions and the general trends in Nusselt number were well captured, however, the magnitude was underpredicted.

Yang and Abdalla ${ }^{14}$ studied an isothermal plate with LES using a dynamic sub-grid-scale model to investigate the secondary instability of a transitional separation bubble. Results presented strong evidence that the elliptical instability was solely responsible for the deformation and three-dimensional breakdown of the Kelvin-Helmholtz instabilities. Langari and Yang ${ }^{15}$ used LES to study the transitional separated flow over a flat plate with semi-circular leading edge. Their analysis showed a change in the primary instability from the Kelvin-Helmholtz instability to a bypasslike transition at elevated freestream turbulence levels. Li and Yang ${ }^{16}$ extended the previous study by assessing the separated transition under a pressure gradient and freestream turbulence. At low freestream turbulence the pressure gradient bypasses the secondary instability in which coherent two-dimensional rolls distort and breakdown into three dimensional structures. Numerical resolution and sub-grid scale turbulent closures for LES were assessed for separated and reattached flows by Cimarelli et al. ${ }^{17}$ using DNS data. Zonal Detached Eddy Simulations (ZDES) were used 
by Pain et al. ${ }^{18}$ for assessing the large scale dynamics of separating and reattaching axisymmetric flow where large scale helical structure and intermediate structures are shown with a Fourier and Dynamic Mode Decomposition (DMD) analysis.

LES has become more practical and accessible within the study of engineering flows with the increase in computational power. Its ability to resolve large-scale eddies and fluctuations, while modelling the small dissipating eddies, has been able to provide accuracy in a range of flows, such as effusion cooling ${ }^{19}$ and jet nozzles ${ }^{20}$. For the foreseeable future, LES is too expensive for design purposes; however, it can unveil a greater understanding of separated and reattached flow and its heat transfer. This can provide practical modelling advice within lower fidelity modelling.

Reynolds-Averaged Navier-Stokes (RANS) calculations are commonly used within industry for flow and heat transfer problems because of their efficiency and low-cost over complex geometries. Formulation of the RANS transport equations are achieved using the Reynolds averaging procedure. Instantaneous quantities are decomposed into a time-mean and fluctuating components. Decomposed quantities are introduced over the transport equations and time-averaged to provide the steady transport equations. The resulting momentum and energy transport equations, Eq. 1 and Eq. 2 respectively, are solved in addition to the continuity equation. As a result of the Reynolds averaging procedure over the non-linear terms, two correlation terms arise: the Reynolds stresses, $\overline{u_{i}^{\prime} u_{j}^{\prime}}$, and the turbulent heat flux $\overline{u_{j}^{\prime} T}$, reflecting the turbulent transport of momentum and heat respectively.

$$
\begin{gathered}
\frac{\partial \rho U_{i}}{\partial t}+\frac{\partial \rho U_{i} U_{j}}{\partial x_{j}}=-\frac{\partial p}{\partial x_{i}}+\frac{\partial}{\partial x_{j}}\left(2 \mu S_{i j}^{*}-\rho \overline{u_{i}^{\prime} u_{j}^{\prime}}\right) \\
\frac{\partial \rho h_{0}}{\partial t}+\frac{\partial \rho U_{j} h_{0}}{\partial x_{j}}=\frac{\partial}{\partial x_{j}}\left(\frac{c_{p} \mu}{\operatorname{Pr}} \frac{\partial T}{\partial x_{j}}+c_{p} \rho \overline{u_{j}^{\prime} T^{\prime}}\right)+\frac{\partial}{\partial x_{j}}\left(u_{i}\left[2 \mu S_{i j}^{*}-\rho \overline{u_{i}^{\prime} u_{j}^{\prime}}\right]\right)
\end{gathered}
$$

Closure of the Reynolds stresses are commonly achieved using the Boussinesq hypothesis ${ }^{21}$ for two-equation turbulence models. This constitutive relation is used with two-equation turbulence models and is described in its incompressible form in Eq. 3. Formulation of the eddy viscosity, $v_{t}$, is achieved using transport equations to describe some length and time scale of the turbulence. Non-linear constitutive relations aim to improve upon the deficiencies of the Boussinesq hypotheses. Speziale ${ }^{22}$ formed a non-linear relation, for the Reynolds stresses, and improved numerical predictions of fully developed duct flow, capturing the secondary flow features that are unseen when using the Boussinesq hypothesis. Reynolds Stress Models (RSM) offer a transport equation 
for each unique Reynolds stress component and a seventh to describe the dissipation, but still rely on closures to describe more unknown correlations. These models often suffer from bad numerical convergence and stability over complex geometries.

$$
\overline{u_{i}^{\prime} u_{j}^{\prime}}=\frac{2}{3} \delta_{i j} k-2 v_{t} S_{i j}
$$

Explicit Algebraic Reynolds Stress Models (EARSM) form a closure for the Reynolds stresses explicitly from the parameters of the mean flow for two-equation turbulence models. Pope ${ }^{23}$ proposed ten tensor groups for this relation and an implicit production to dissipation ratio for two-dimensional mean flows. Wallin and Johansson ${ }^{24}$ form an EARSM model with a near-wall treatment using a van-Driest function. For two-dimensional flows the anisotropy of the Reynolds stresses is evaluated using Eq. 4, where the first term of the equation is akin to the anisotropy of the Boussinesq hypothesis.

$a_{i j}=f_{1} \beta_{1} S_{i j}+\left(1-f_{1}^{2}\right) \frac{3 B_{2}-4}{\max \left(I I_{S}, I I_{S}^{e q}\right)}\left(S_{i j}^{2}-\frac{1}{3} I I_{S} \delta_{i j}\right)+\left[f_{1}^{2} \beta_{4}-\left(1-f_{1}^{2}\right) \frac{B_{2}}{2 \max \left(I I_{S}, I I_{S}^{e q}\right)}\right]\left(S_{i k} \Omega_{k j}-\Omega_{i k} S_{k j}\right)$

The Reynolds-averaged energy equation requires the closure of the turbulent heat flux vector, $\overline{u_{i}^{\prime} T^{\prime}}$. A simple Gradient Diffusion Hypothesis (GDH), expressed by Eq. 5, is commonly implemented in RANS codes. The model aligns the turbulent heat flux vector to the mean temperature gradient of the flow. The turbulent diffusivity, $\alpha_{t}$, which controls the additional diffusion of the turbulence, is isotropic, described by the flows eddy viscosity and the turbulent Prandtl number, $P r_{t}$. These assumptions are not true for complex flows where the size of energy-containing eddies are comparable to or greater than the length over which the thermal gradient changes significantly ${ }^{25}$. Turbulent flat plate correlations in the log-law region provide a turbulent Prandtl, $P r_{t}$, number of $0.85^{26}$. The value has been debated for other flows and a spatially varying turbulent Prandtl number, within the boundary layer, can improve the heat transfer results ${ }^{27}$.

The Generalised GDH (GGDH) is an algebraic closure introduced by Daly and Harlow ${ }^{28}$. For the turbulent heat flux closure the equation is shown in Eq. 6. Inclusion of the Reynolds-stress terms introduces anisotropy into the turbulent diffusivity, improving the accuracy of the predictions where an accurate description of the Reynolds stress field is provided. A model constant, $c_{\theta}$, replaces the turbulent Prandtl number of the GDH. A higher order closure can be achieved using the quadratic products of the Reynolds stresses for the High-Order GGDH (HOGGDH) closure ${ }^{29}$ 
presented in Eq. 7 and extends upon these diffusion hypothesis methods. A transport equation of the turbulent heat flux vector can also be found, as performed by Le Ribault and Friedrich ${ }^{30}$, but these increasing complexities are uncommon within commercial CFD codes.

$$
\begin{aligned}
& \overline{u_{i}^{\prime} T^{\prime}}=-\alpha_{t} \frac{\partial \bar{T}}{\partial x_{i}}=-\frac{v_{t}}{P r_{t}} \frac{\partial \bar{T}}{\partial x_{i}} \\
& \overline{u_{i}^{\prime} T^{\prime}}=-c_{\theta} \tau_{t} \overline{u_{i}^{\prime} u_{j}^{\prime}} \frac{\partial \bar{T}}{\partial x_{j}} \\
& \overline{u_{i}^{\prime} T^{\prime}}=-c_{\theta} \tau_{t} \frac{\overline{u_{i}^{\prime} u_{k}^{\prime}} \overline{u_{k}^{\prime} u_{j}^{\prime}}}{k} \frac{\partial \bar{T}}{\partial x_{j}}
\end{aligned}
$$

GDH and HOGGDH closures are assessed by Ling et al. ${ }^{31}$ for the turbulent scalar flux in discrete hole film cooling flow. Although the HOGGDH captures anisotropy in the scalar flux, the magnitude relies upon the model coefficient. Forward propagation to the energy equation showed the effects upon surface cooling, where the best results were seen from using the GDH with an LES derived isotropic turbulent diffusivity. Abe and Suga ${ }^{29}$ study the turbulent scalar flux models in differing channel flows. Within shear-free flow regions, the GGDH closure produced a reasonable approximation of the turbulent scalar flux. However, close to the wall the GGDH did not provide a correct ratio of normal to streamwise turbulent scalar flux, whereas the HOGGDH method showed improved predictions.

Explicit Algebraic Scalar Flux Models (EASFM) formulate a closure for turbulent scalar flux in an approach that is similar to the EARSM formulation. Wikström et al. ${ }^{32}$ propose a model which is formed by applying an equilibrium condition in the normalised scalar flux transport equation. The closure requires additional transport models to obtain the half scalar variance and its dissipation rate to close the system of equations, alongside accurate Reynolds stress predictions from either an EARSM or RSM.

In this paper, LES is used to achieve accurate predictions of heat transfer and turbulent flow for the heated blunt plate flow, shown in Fig. 1. An LES informed Turbulent Transport (LiTT) procedure solves the $k-\omega$ SST specific dissipation rate transport equation over an LES time-averaged field. The results are then used to assess the deficits within closures of the Reynolds stresses and the turbulent heat flux. Improved predictions are sought for the HOGGDH closure and an optimal coefficient is studied over the separated and reattached flow regime to improve the surface heat transfer predictions. 


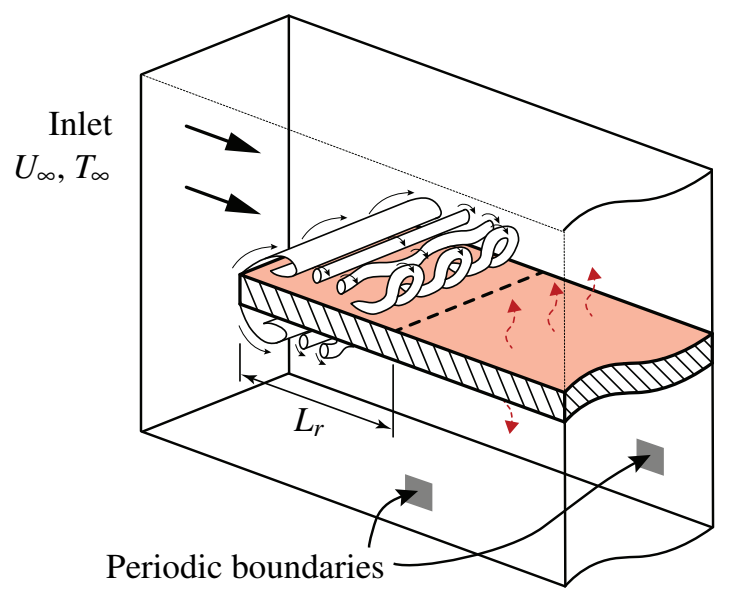

FIG. 1: Heated blunt plate geometry and computational domain.

\section{NUMERICAL PROCEDURES}

\section{A. Eddy-Resolving Simulations}

Simulations were performed using the rhoPimpleFoam solver in OpenFOAM Version $5.0^{33}$. OpenFOAM is an open source CFD tool box that rivals common commercial packages with an abundance of turbulence modelling solutions and has been widely used in research communities. It has developed traction in a broad range of fields such as jet flows $\mathrm{s}^{34,35}$, combustion flows ${ }^{36}$ and two-phase flow modelling ${ }^{37}$, amongst many others.

The conservation equations for continuity, momentum and energy are solved in the following forms respectively:

$$
\begin{gathered}
\frac{\partial \bar{\rho}}{\partial t}+\frac{\partial \bar{\rho} \tilde{u}_{i}}{\partial x_{i}}=0 \\
\frac{\partial \bar{\rho} \tilde{u_{i}}}{\partial t}+\frac{\partial \bar{\rho} \tilde{u}_{i} \tilde{u_{j}}}{\partial x_{j}}=-\frac{\partial \bar{p}}{\partial x_{i}}+\frac{\partial}{\partial x_{j}}\left(2 \mu \tilde{S}_{i j}^{*}-\bar{\rho} \tau_{i j}^{s g s}\right) \\
\frac{\partial \bar{\rho} \tilde{h_{0}}}{\partial t}+\frac{\partial \bar{\rho} \tilde{u_{j}} \tilde{h_{0}}}{\partial x_{j}}=\frac{\partial}{\partial x_{j}}\left(\bar{\rho} \alpha \frac{\partial \tilde{h}}{\partial x_{j}}-\bar{\rho} q_{j}^{s g s}\right) \\
\tau_{i j}=-2 v_{s g s} \tilde{S}_{i j}^{*} \\
\tilde{S}_{i j}^{*}=\frac{1}{2}\left(\frac{\partial \tilde{u}_{i}}{\partial x_{j}}+\frac{\partial \tilde{u}_{j}}{\partial x_{i}}\right)-\frac{1}{3} \frac{\partial \tilde{u}_{k}}{\partial x_{k}}
\end{gathered}
$$


Sub-Grid Scale (SGS) stresses are modelled using the Wall-Adaptive Local Eddy-viscosity (WALE) model $^{38}$. The SGS stresses are described by a modelled SGS eddy viscosity, $v_{s g s}$, and the mesh resolved traceless strain rate tensor, $\tilde{S}_{i j}^{*}$. The model provides correct near-wall scaling of the eddy viscosity with $y^{3}$ without the requirement of a dynamic formulation. WALE has been successfully used within conjugate heat transfer simulations of cooled turbine blades ${ }^{39}$, the flow and heat transfer of two cylinders in tandem ${ }^{40}$ and rib-roughened internal turbine blade cooling channels ${ }^{41}$. The model has also proved successful in reproducing transition from laminar instabilities ${ }^{38}$. However, WALE predictions of the SGS viscosity are dependant on limitations of the model coefficient $c_{w}$. Values of 0.5 provide success in homogeneous isotropic decaying turbulence $^{38}$ while a value of 0.325 has become common practice in complex flows providing suitable diffusion ${ }^{42}$, although the model results remain relatively insensitive to the coefficient ${ }^{43,44}$.

$$
\begin{gathered}
v_{s g s}=\left(C_{w} \Delta\right)^{2} \frac{\left(S_{i j}^{d} S_{i j}^{d}\right)^{3 / 2}}{\left(\bar{S}_{i j} \bar{S}_{i j}\right)^{5 / 2}+\left(S_{i j}^{d} S_{i j}^{d}\right)^{5 / 4}} \\
\left.S_{i j}^{d}=\frac{1}{2}\left(\bar{g}_{i j}^{2}+\bar{g}_{j i}^{2}\right)\right)-\frac{1}{3} \delta_{i j} \bar{g}_{k k}^{2} \\
\bar{g}_{i j}=\frac{\partial \bar{u}_{i}}{\partial x_{j}}
\end{gathered}
$$

The SGS viscosity is obtained from Eq. 13 where, $\Delta$, is the cube root of the local cell volume and the model coefficient, $C_{w}$, of 0.325 is selected. The definition of $S_{i j}^{d}$ is shown in Eq. 14 formings the traceless symmetric component of the square of the velocity gradient tensor, $\bar{g}_{i j}$.

$$
\begin{gathered}
q_{j, s g s}=-\alpha_{s g s} \frac{\partial h}{\partial x_{j}} \\
\alpha_{s g s}=\frac{v_{s g s}}{P r_{s g s}}
\end{gathered}
$$

SGS heat fluxes are obtained from Eq. 16 where the SGS turbulent diffusivity is defined by Eq. 17. The SGS Prandtl number, $P r_{s g s}$, was set to 0.4, as used in LES simulations by Schindler et al. ${ }^{45}$ and Eidson ${ }^{46}$ and recommended by Grötzbach and Wörner ${ }^{47}$. Although other simulations have been known to use greater values of $\operatorname{Pr}_{s g s}{ }^{13,48}$. Initial tests investigated the sensitivity of surface heat transfer results to $P r_{\text {sgs }}$. Values of 0.4 to 1.0, as used in Pallares and Davidson's ${ }^{49}$ channel flow case, showed no significant difference for the mesh used. 
TABLE I: Physical parameters to replicate experimental conditions of Ota and Kon ${ }^{1}$.

\begin{tabular}{lr}
\hline \hline Plate Thickness & $H=20 \mathrm{~mm}$ \\
Upstream Velocity & $U_{\infty}=27 \mathrm{~m} / \mathrm{s}$ \\
Upstream Temperature & $T_{\infty}=300 \mathrm{~K}$ \\
Outlet Pressure & $p=101325 \mathrm{~Pa}$ \\
Wall Heat Flux & $q=668.28 \mathrm{~W} / \mathrm{m}^{2}$ \\
Reynolds Number & $R_{H}=21600$ \\
\hline \hline
\end{tabular}

Coupling of the continuity and momentum equations are achieved iteratively using the PISO algorithm. Time stepping is discretised using a first-order implicit Euler scheme with a time step providing a maximum $C F L$ of 0.8 to achieve time accurate and numerically stable results. Convective fluxes are discretised using a central scheme with an upwind bias to achieve second-order spatial accuracy. The discretisation of diffusive fluxes is achieved using a fully central scheme.

Boundary conditions are used to replicate the experimental conditions of Ota and $\mathrm{Kon}^{1}$ and achieve the conditions outlined in Table I. The Reynolds number is characterised by the plate thickness, $H$. No-slip wall conditions are applied to the top, bottom and leading-edge surfaces of the plate with a wall-normal pressure gradient of zero. A constant heat flux boundary condition applies $668.28 \mathrm{~W} / \mathrm{m}^{2}$ to the top and bottom surfaces replicating the experimental validation case. A velocity inlet is used with $U_{\infty}=27 \mathrm{~m} / \mathrm{s}$ and $T_{\infty}=300 \mathrm{~K}$ with pressure gradient set to zero. The pressure outlet boundary condition defines a pressure of $101325 \mathrm{~Pa}$. Periodic conditions are applied to the spanwise constrained boundaries, shown in Fig. 1, simulating an infinite span and permitting vortical structures to pass from one side to the other. Slip walls are applied to the top and bottom boundaries to replicate the blockage conditions of the experimental case.

A tetrahedral octree meshing strategy is used to provide shear layer and near-wall refinements without the high-aspect ratio, skewed cells and unnecessary far-field refinements associated with structured techniques. Results are obtained using a 16 million cell mesh. Effects of grid resolution were assessed with a 5, 11 and 16 million cell mesh and no difference was observed in the timeaveraged surface heat transfer between the 11 and 16 million cell meshes. Near-wall cell height was selected to achieve a $\Delta y^{+}$of less than 1 while streamwise and spanwise spacing were selected to achieve $\Delta x^{+}$and $\Delta y^{+}$of less than 25 . Volumetric refinements were used in the recirculation region (Fig. 2a), the shear layer and near-wall regions (Fig. 2b) to appropriately resolve the 


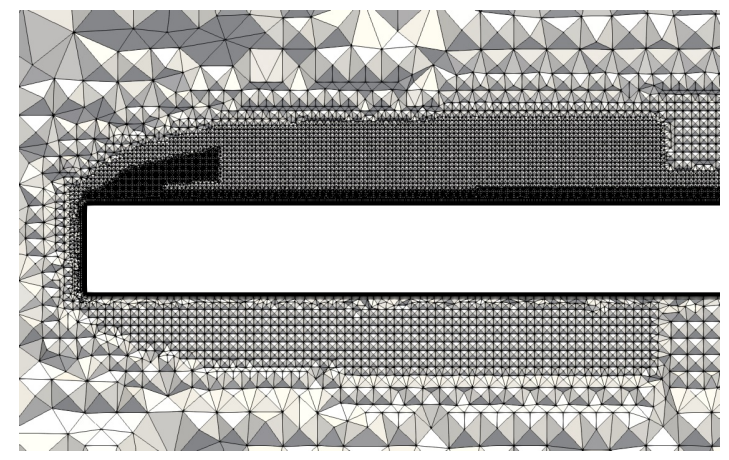

(a)

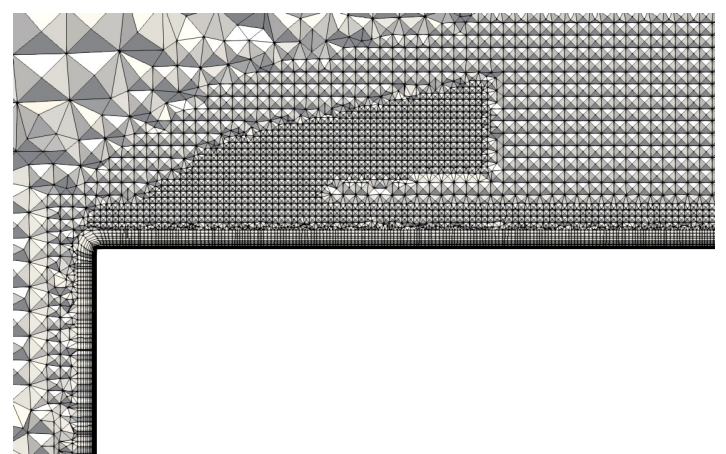

(b)

FIG. 2: Tetrahedral octree meshing strategy showing highlighting locally refined regions. (a) Recirculation refinements and (b) shear layer and near-wall refinements.

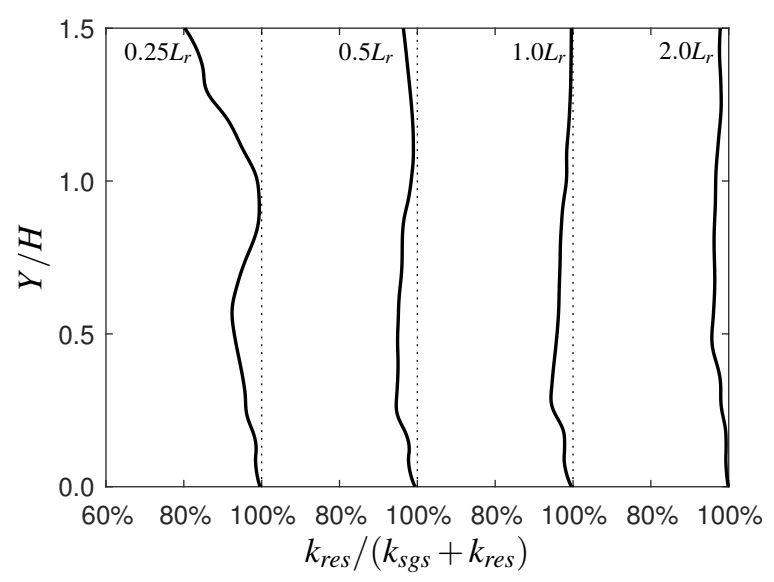

FIG. 3: Comparison of resolved to sub-grid scale turbulent kinetic energy.

large scale motions and three-dimensional vortical structures. The bottom side of the plate was coarsened to reduce cell quantity while retaining any flow field coupling between top and bottom surfaces. The numerical results shown throughout this paper will feature the top side of the plate only. For the top side of the plate, the mesh resolves more than $90 \%$ of the turbulent kinetic energy as shown by the profiles in Figure 3. Aft of the recirculation centre, 95\% of the turbulent kinetic energy is resolved. Analysis within this paper will only consider the resolved fluctuations of the flow as the sub-grid scale contributions are shown to be small. 


\section{B. LES informed Turbulent Transport (LiTT) Analysis}

RANS closures are investigated using the high-fidelity LES data in an a priori analysis. Timeaveraged results from the LES provides well-resolved Reynolds stress and turbulent heat flux fields. A RANS simulation can be performed and the modelled turbulent transport terms presented; however, the flow fields are inherently different because of the modelling discrepancies and direct comparisons are not possible to experimental or eddy-resolving datasets. Raiesi et al. ${ }^{50}$ pursue an array of solutions for assessing turbulence model behaviour from DNS and LES datasets in an a prior analysis and previous work by Parneix et al. ${ }^{51}$ used similar a priori analysis of second moment closures and the importance of these types of studies are discussed by Durbin ${ }^{52}$. Weatheritt and Sandberg ${ }^{53}$ propose a solution for extracting a dissipation rate from high-fidelity datasets to aid machine learning model improvements to Reynolds stress field predictions in RANS simulations.

The time-averaged LES field is frozen and the resolved turbulent kinetic energy, $k$, is calculated. The transport equation for the $k-\omega \mathrm{SST}^{54}$ specific dissipation rate, $\omega$, is solved iteratively over the frozen LES field (Eq. 18) where the eddy-viscosity, $v_{t}$, is modelled by Eq. 19 and the constants are consistent with those from Menter ${ }^{55}$. The approach enables functional predictions of turbulent dissipation and eddy viscosity that reflect the values that a RANS would calculate if the flow field and turbulent kinetic energy were identical to the LES. With this information, the Reynolds stresses and the turbulent heat flux quantities, modelled by RANS closures, can be assessed. Hence, this $a$ priori approach has been coined as an LES informed Turbulent Transport (LiTT) analysis.

$$
\begin{gathered}
\frac{\partial \rho \omega}{\partial t}+\frac{\partial \rho u_{j} \omega}{\partial x_{j}}=\frac{\gamma}{v_{t}} P_{k}-\beta \rho \omega^{2}+\frac{\partial}{\partial x_{j}}\left[\left(\mu+\sigma_{\omega} \mu_{t}\right) \frac{\partial \omega}{\partial x_{j}}\right]+2\left(1-F_{1}\right) \frac{\rho \sigma_{\omega 2}}{\omega} \frac{\partial k}{\partial x_{j}} \frac{\partial \omega}{\partial x_{j}} \\
v_{t}=\frac{a_{1} k}{\max \left(a_{1} \omega, \Omega F_{2}\right)}
\end{gathered}
$$

The LiTT analysis is firstly used to identify differences in the turbulent anisotropy resolved by the LES to that described by the Boussinesq hypothesis (Eq. 3), where $v_{t}$ is taken from the LiTT and the strain-rate, $\bar{S}_{i j}$, is from the time-averaged frozen LES field. Comparisons are furthered by analysing the EARSM formulation with near-wall treatment of Wallin and Johansson ${ }^{24}$ (Eq. 4) and their enhanced predictions of the Reynolds stress field and anisotropy. 
Secondly, the analysis is used to assess functional discrepancies in the turbulent heat flux closures. The GDH (Eq. 5), commonly used within commercial CFD packages, takes the eddyviscosity described by the LiTT analysis with the commonly used coefficient $P r_{t}=0.85$. The GGDH (Eq. 6) and HOGGDH (Eq. 7) models are assessed with LiTT where the turbulent time scale is defined by $\tau=k / \varepsilon=1 /\left(\beta^{*} \omega\right)$ and the Reynolds stress fields are initially taken from the LES to demonstrate their improvements when a realistic Reynolds stress field is provided. The EASFM of Wikström et al. ${ }^{32}$ is then investigated with the LiTT for the discussed flow, showing comparisons to the diffusion hypothesis models. The formulation of the EASFM is taken from Wilksröm et al. ${ }^{32}$ where the scalar term has been replaced by the temperature and shown in Eq. 20.

$$
\overline{u_{i}^{\prime} T^{\prime}}=-\left(1-c_{\theta 4}\right) A_{i j}^{-1}\left(a_{j k}+\frac{2}{3} \delta_{j k}\right) \frac{k^{2}}{\varepsilon} \frac{\partial \bar{T}}{\partial x_{i}},
$$

where for two-dimensional mean flow,

$$
\boldsymbol{A}^{-1}=\frac{G \boldsymbol{I}-\left(c_{S} \boldsymbol{S}+c_{\Omega} \Omega\right)}{G .^{2}-\frac{1}{2} Q_{1}}-\left(\frac{G}{G^{2}-\frac{1}{2} Q_{1}}-\frac{1}{G}\right)\left(\boldsymbol{I}-\boldsymbol{I}_{2 D}\right)
$$

and,

$$
\begin{gathered}
Q_{1}=c_{S}^{2} I_{S}+c_{\Omega}^{2} I I_{\Omega}, \\
G=\frac{1}{2}\left(2 c_{\theta 1}-1-\frac{1}{r}+\frac{P_{k}}{\varepsilon}\right) .
\end{gathered}
$$

The two-dimensional invariants of the mean strain rate and vorticity are defined as:

$$
I I_{S}=\operatorname{tr}\left\{S^{2}\right\}, \quad I I_{\Omega}=\operatorname{tr}\left\{\Omega^{2}\right\}
$$

The time scale ratio, $r$, defines the ratio of scalar to turbulent time scale, where the scalar time scale is equal to the ratio of half-scalar variance to scalar dissipation rate. For the RANS approaches studied, this ratio is unclosed as the scalar variance and dissipation rate are not available. Modifications would require additional transport equations for these two properties or alternatively an explicit relation. For the current analysis, the high fidelity LES dataset is used to describe the 
time scale ratio by assuming it is equal to ratio of the integral time scale of the temperature autocorrelation and streamwise velocity autocorrelation across the flow. Finally, Constants within the model are defined as $c_{\theta 4}=0, c_{S}=c_{\Omega}=1$ and $c_{\theta 1}$ is set to $1.6(r+1) / r$. Strain rates, $\boldsymbol{S}$, and vorticity, $\Omega$, are normalised with the turbulent time scale, $\tau_{t}$.

\section{RANS k- $\omega$ SST Analysis}

A RANS solution using the two-equation $k-\omega$ SST turbulence model is performed for the purpose of comparing first order statistics of the flow. The implementation of the model used is coherent with the LiTT analysis, but the transport equations for continuity, momentum and energy are solved with the turbulent kinetic energy and specific dissipation rate. Closure of the Reynolds stress terms are achieved using the Boussinesq hypothesis constitutive relation and the turbulent heat flux is closed with the GDH.

A two-dimensional structured mesh with $y^{+}<1$ and a clustering of cells focussed around the recirculation region are used to resolve the flow. The meshing strategy involved refinement of the grid until the surface Nusselt number distribution no longer changed with additional refinement. A symmetry plane along the plate centreline was used to replicate the symmetry of the mean flow over the plate.

Other turbulence models were investigated in a preliminary study, but did not provide adequate heat transfer solutions. Thus the selection of the $k-\omega$ SST model provides a suitable comparison to the LES and a baseline for further improvements.

TABLE II: Analysis methods and their descriptions.

\begin{tabular}{ll}
\hline \hline Method & Description \\
\hline LES & Pure LES results using the WALE sub-grid scale model. \\
$k-\omega$ SST & Full RANS computation with the $k$ - $\omega$ SST turbulence model. \\
LiTT-BH & LiTT of the Boussinesq hypothesis modelled Reynolds stresses. \\
LiTT-EARSM & LiTT of the EARSM modelled Reynolds stresses with near-wall enhancement. \\
LiTT-GDH & LiTT of GDH modelled turbulent heat flux. \\
LiTT-GGDH & LiTT of GGDH modelled turbulent heat flux. \\
LiTT-HOGGDH & LiTT of HOGGDH modelled turbulent heat flux. \\
LiTT-EASFM & LiTT of EASFM modelled turbulent heat flux. \\
\hline \hline
\end{tabular}




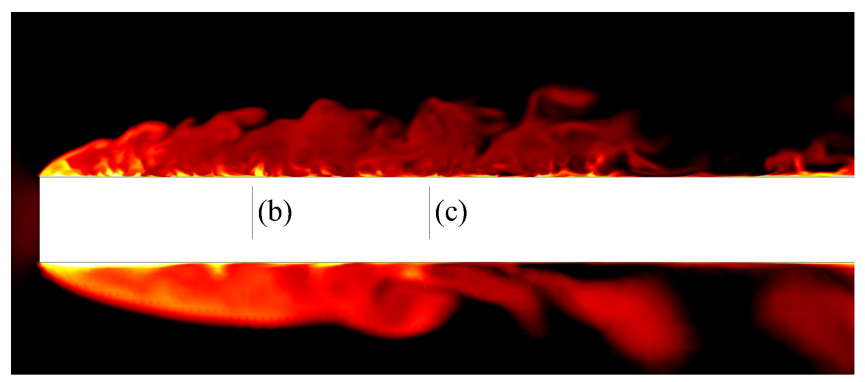

(a) Slice $Z=0$
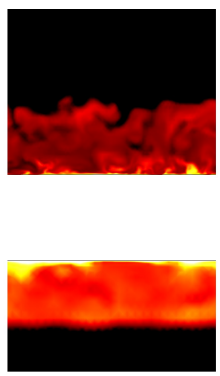

(b) Slice $X=2.5 H$
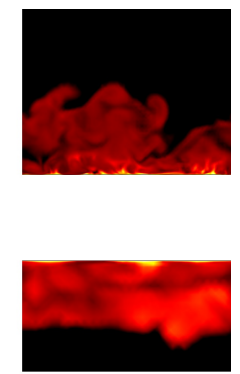

(c) Slice $X=L_{r}$

$\begin{array}{lllllllll}300 & 301 & 302 & 303 & 304 & 305 & 306 & 307 & 308\end{array}$

$T(K)$

FIG. 4: Instantaneous temperature across (a) spanwise slice at $Z=0$ and streamwise slices at (b) $X=2.5 H$ and (c) $X=L_{r}$. "(Multimedia view)"

\section{RESULTS AND DISCUSSIONS}

Results and discussions will be presented in three parts. Firstly, the success of the LES using WALE SGS modelling will be shown by comparison to experimental results. Secondly, an assessment of the turbulence anisotropy is presented and the deficits of Reynolds stresses modelled by Boussinesq hypothesis in RANS simulations are studied with the LiTT analysis. Finally, the LiTT approach will evaluate RANS closures to the turbulent heat flux vector to identify the best approach for RANS simulations of complex turbulent forced convection flows. Table II presents the method and descriptions that will be used throughout the paper. Legends provided in the figures will remain consistent with the table to aid the reader.

\section{A. Flow Field}

Figure 4 "(Multimedia view)" presents an instantaneous snap shot of the temperature field, showing the influence of turbulent fluctuations and vortex structures upon the thermal state of the flow. The initial two-dimensional roll up of the shear layer is visible in the temperature field with structures consistent with Kelvin-Helmholtz instabilities. The three-dimensional unsteadiness of the turbulent flow is presented in the streamwise slices of Figure 4b "(Multimedia view)" and $4 c$ "(Multimedia view)". At the reattachment location, the presented temperature field shows an enhancement in the turbulent mixing with the presence of three-dimensional vortical structures of varying sizes. The coarsened underside of the plate and its effect upon the thermal field identifies 


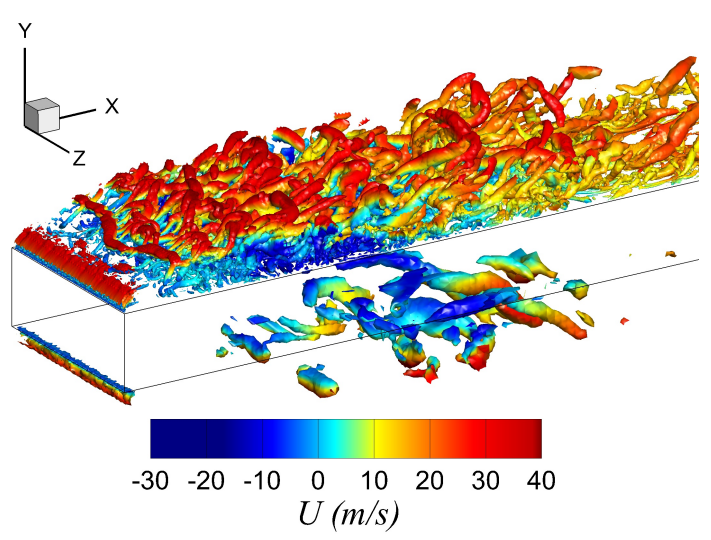

FIG. 5: Vortex structures within the recirculation and reattachment regions using the $\lambda_{2}$ criterion. "(Multimedia view)"

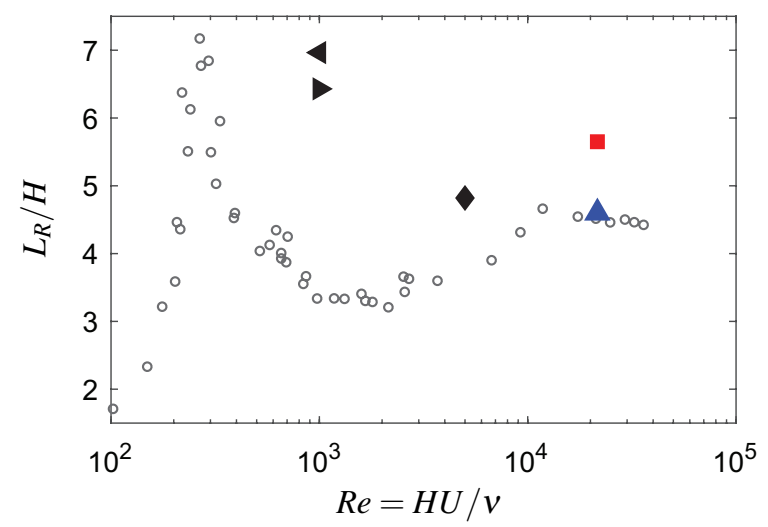

FIG. 6: Reattachment location. LES ( $\bullet$ ), $k-\omega \operatorname{SST}(\bullet)$, Ota and Yanaoka ${ }^{10}(\bullet)$, Tafti and $\operatorname{Vanka}^{11}(\bullet)$, Yanaoka et al. ${ }^{12}(\bullet)$ and Ota et al. ${ }^{56}(\bullet)$.

the expected under-resolved flow, but presents the large scale disturbances that could drive any coupling between the flow above and below the plate.

The aforementioned turbulent behaviours are presented with the $\lambda_{2}$ criterion in Figure 5 "(Multimedia view)". Initial instabilities in the shear layer show a short two-dimensional spanwise vortex, which breaks down into three dimensional hairpin structures. Hairpin structures elongate as the flow develops across the reattachment location and downstream. Within the recirculation region, very small turbulent structures are unveiled within the large scale recirculating flow.

Figure 6 shows predictions of time-averaged reattachment length, $L_{r}$, with Reynolds number, comparing previous numerical studies ${ }^{10-12}$ and the present studies with the experimental correlation of Ota et al. ${ }^{56}$. The time-averaged reattachment length is defined as the distance from the plate leading-edge to the location of zero time-averaged skin friction coefficient. Good agree- 


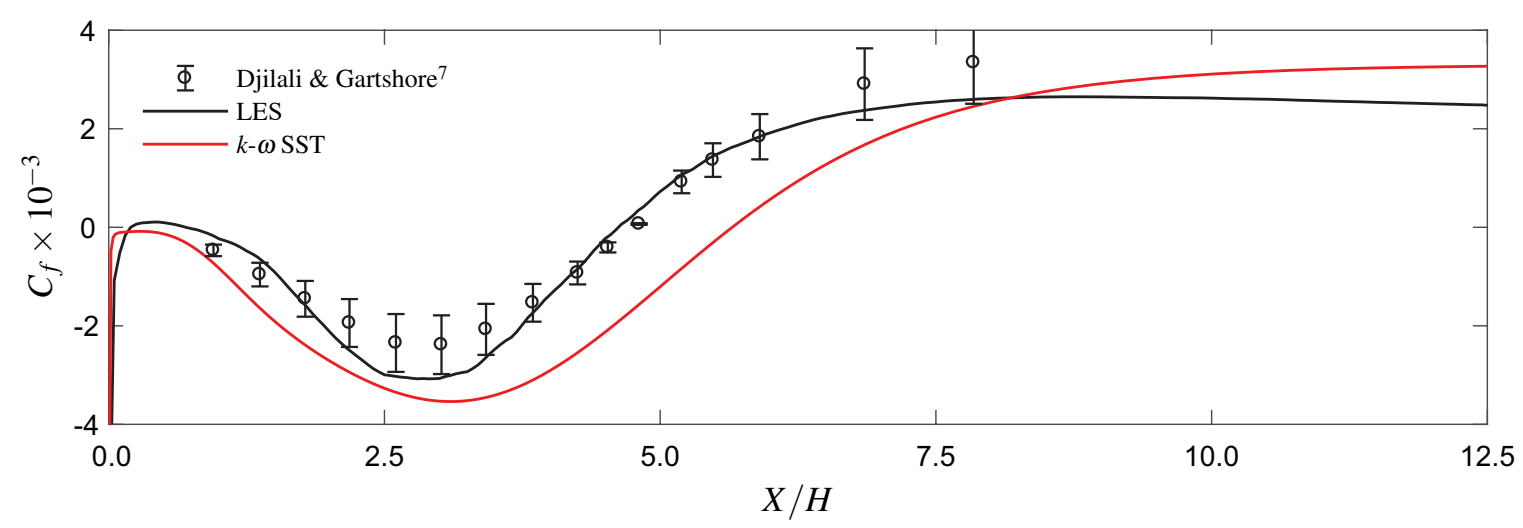

FIG. 7: Comparison of friction coefficient calculated to pulsed wall probe experimental results.

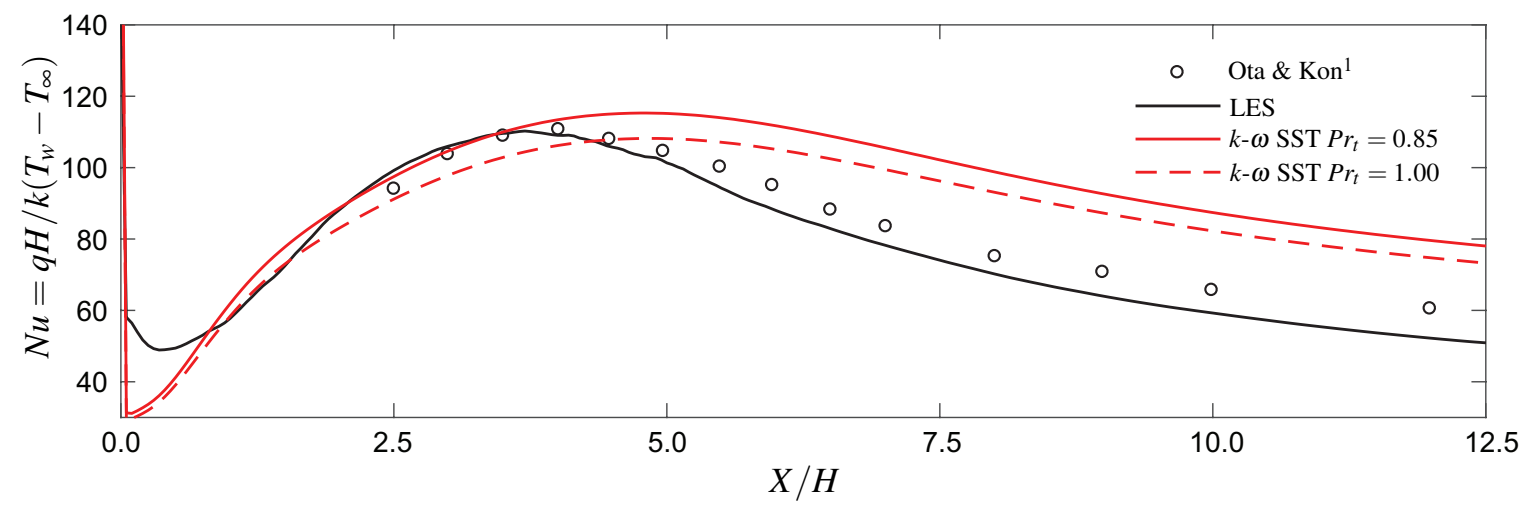

FIG. 8: Time and spanwise-averaged Nusselt number compared to the experimental case of Ota and $\mathrm{Kon}^{1}$ and two-dimensional $k$ - $\omega$ SST solutions.

ment is shown the to experimental correlations of Ota et al. ${ }^{56}$. A reattachment length of $4.6 \mathrm{H}$ is predicted by the present LES. The SST RANS solution provides a reattachment length of $5.7 H$ showing an overestimation of $24 \%$.

The time-averaged skin friction coefficient, $C_{f}$, is presented in Figure 7. Within the recirculation region, distinguished by the negative value of $C_{f}$, the location of minimum $C_{f}$ is correctly captured. The peak value occurs in the redeveloping region followed by a decreasing value reflecting the development of the boundary layer. Recirculation and redevelopment $C_{f}$ trends are captured by the SST RANS, but offset by the overestimation of the reattachment length while the magnitude of the wall shear stress is over predicted in the recirculation region.

Time-averaged Nusselt number predictions of the present LES are shown to be in good agreement with the experimental case of Ota and $\mathrm{Kon}^{1}$. The peak Nusselt number is present at a location just upstream of the reattachment location, consistent with the long separation bubble observations 


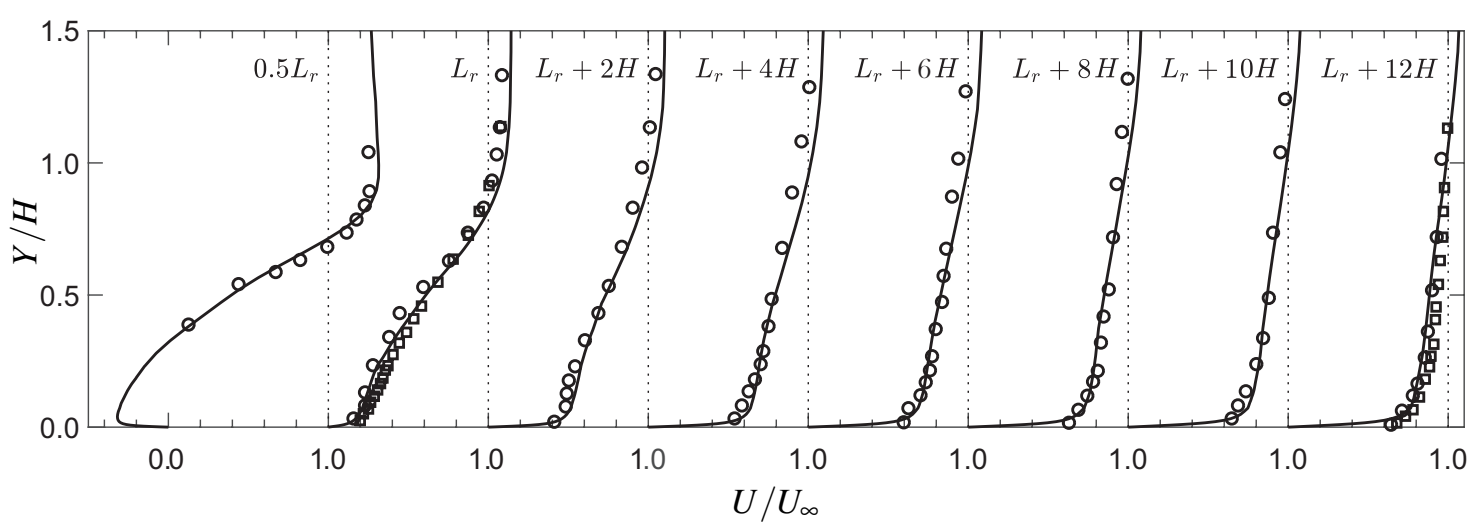

FIG. 9: Non-dimensional velocity profiles over plate. LES (—), Ota and $\operatorname{Kon}^{1}\left({ }^{\circ}\right)$ and Ota and $\operatorname{Kon}^{3}(\mathbf{a})$.

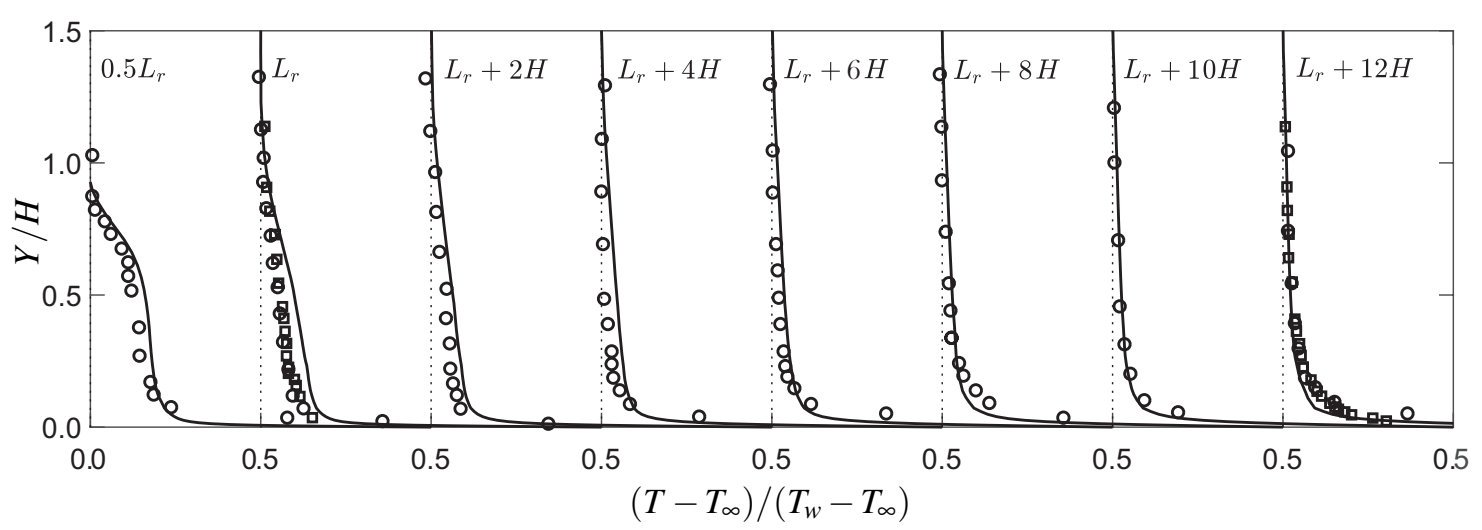

FIG. 10: Non-dimensional temperature profiles over plate. Legend see Figure 9.

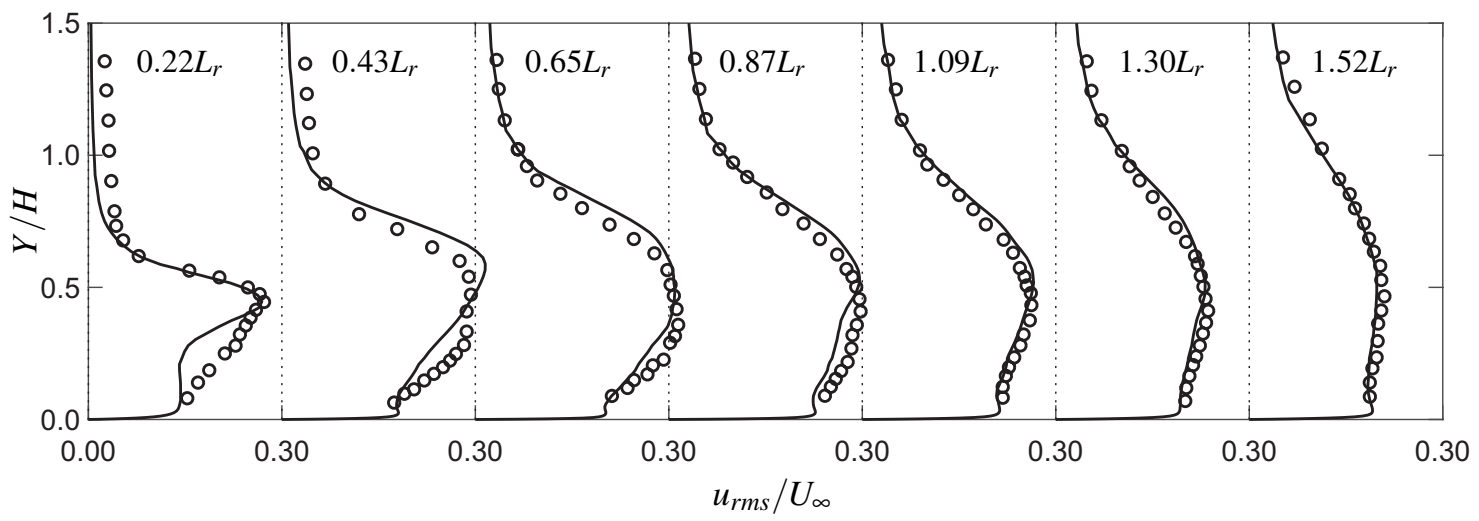

FIG. 11: RMS streamwise velocity development through the recirculation and reattachment region. LES (一) and $\mathrm{PDA}^{7}\left({ }^{\circ}\right)$.

of Sparrow et al. ${ }^{57}$. A small underprediction of the Nusselt number is shown in the redeveloping region. Results of the RANS $k-\omega$ SST solution are shown at two turbulent Prandtl numbers which 
demonstrates the sensitivity of Nusselt number magnitude to the parameter. The RANS $k$ - $\omega$ SST shows an overestimation of the peak Nusselt number location and an overprediction of the magnitude in the redeveloping region. Results from the LES simulation show an improved degree of accuracy in heat transfer predictions over the RANS SST solution.

Non-dimensional velocity profiles are compared in Figure 9 showing the time-averaged flow over the recirculation, reattachment and redeveloping regions. Good agreement is made to the experimental results of Ota and $\operatorname{Kon}^{1}(R e=23400)$ and Ota and $\operatorname{Kon}^{3}(R e=21600)$. Profile positions are normalised by the reattachment length to compare for the shorter recirculation region observed by Ota and Kon ${ }^{1}$. The accurate predictions of the LES show the recirculation bubble height is correctly predicted. Within the redeveloping region, the additional upstream turbulence from the turbulent shear layer, enhances the turbulent fluctuations of the boundary layer flow. The present LES simulation shows good agreement with the influence of this enhancement on the time-averaged profiles observed in the experiment.

Figure 10 compares profiles of non-dimensional time-averaged temperature to the experimental work of Ota and Kon ${ }^{1,3}$ at the Reynolds numbers of 23400 and 25000. General trends, reflecting the increased values within the recirculation region, are well captured. The LES results present a small overprediction in the recirculation and reattachment regions. Unlike the time-averaged velocity field, the temperature field is strongly correlated with the Reynolds number ${ }^{12}$. This observation shows that these profiles have decreasing magnitudes with increasing Reynolds number. The present LES work is consistent within this observation although experimental results show comparative magnitudes.

Figure 11 shows the present LES predictions of the root-mean-square values of streamwise velocity compared to the Pulse Dopler Anemometry (PDA) measurements of Djilali and Gartshore ${ }^{7}$ for their non-heated blunt plate study. For these flow regimes, PDA is a well suited measurement technique, where the fluctuating components are a similar or greater value than their mean components. Following the reattachment location, excellent agreement is shown within the profiles and the enhanced turbulent fluctuations in the redeveloping boundary layer are present. Through the recirculation region, LES predictions closely match the experimental data. Small underpredictions of the root-mean-square (rms) value are present within the first $0.25 \mathrm{H}$ of the profiles of the recirculation region.

The complexity of the turbulent behaviour is observed in the near-wall behaviour of turbulent eddies. Instantaneous streamwise and normal velocities with the flow temperature are presented 


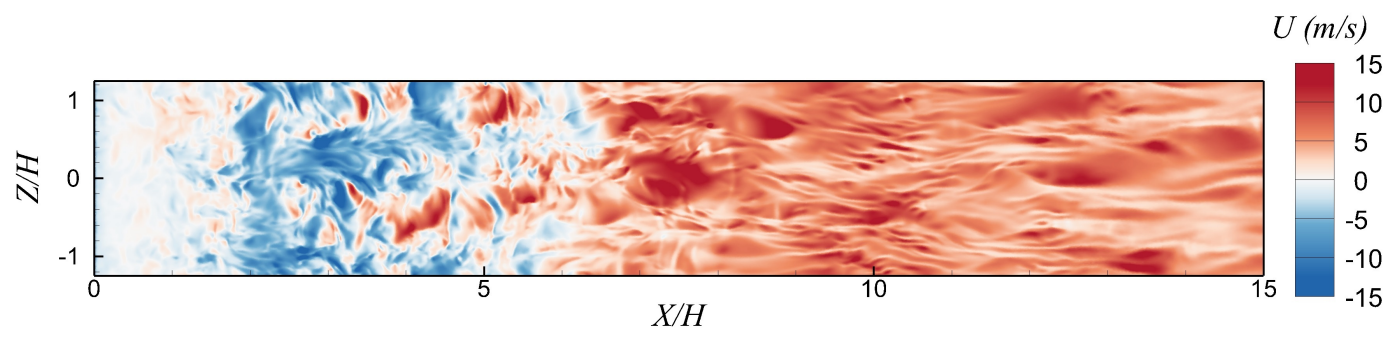

(a) Streawise velocity

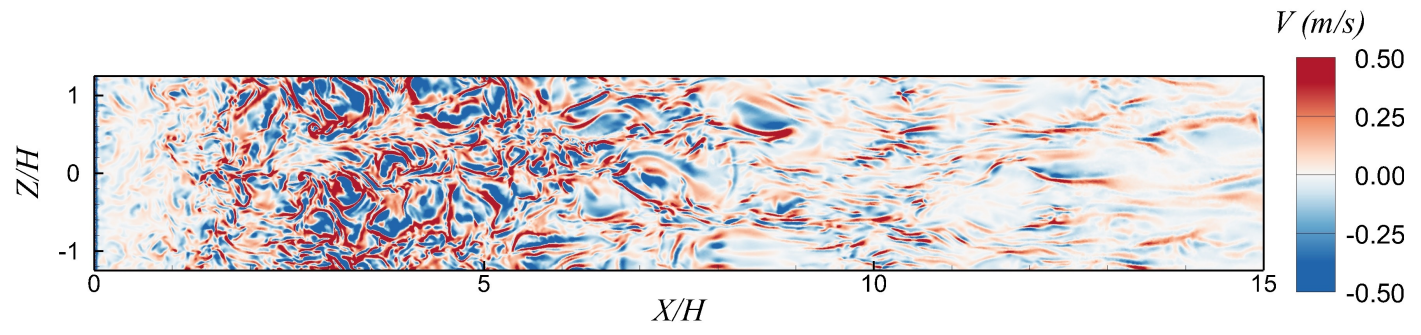

(b) Normal velocity

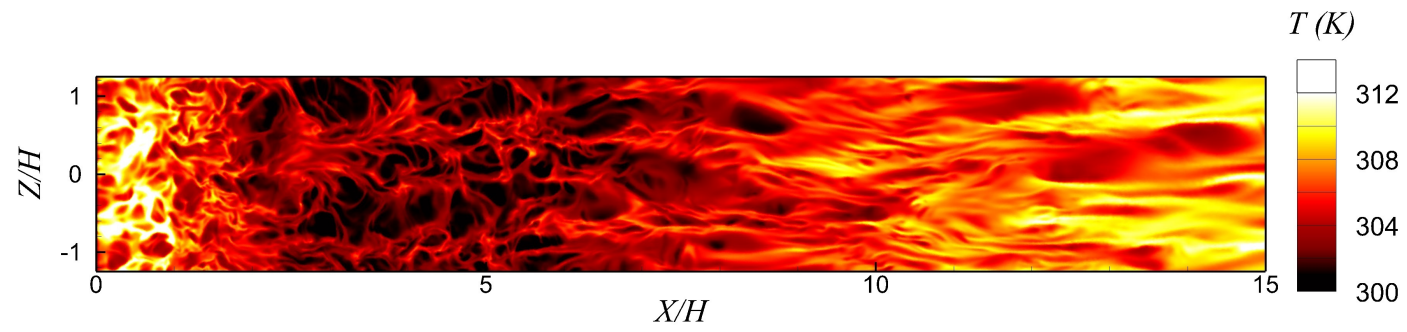

(c) Temperature

FIG. 12: Instantaneous near-wall $\left(y^{+} \approx 10\right)$ contours of (a) streamwise velocity, (b) normal velocity and (c) temperature. "(Multimedia view)"

in Fig. 12 "(Multimedia view)" at a normal plane of $y^{+} \approx 10$. A qualitative coupling is observed between the velocity and temperature fields driven by the turbulent eddies. Within the recirculation and reattachment region, normal velocities (Fig. 12b "(Multimedia view)") present eddies in varying orientations at a range of scales. The eddy structures observed in the normal velocities, reflect structures present in the near-wall temperature field (Fig. 12c "(Multimedia view)") which influence the instantaneous surface heat transfer. Heated fluid is lifted from the surface by the positive normal velocity and cooler fluid is deposited on the other side of the eddies where a negative normal velocity is present. Behind the separation point, the near-wall flow is relatively slow presenting a higher flow residence time in which the local surface heat transfer is reduced. Downstream of the reattachment region, the hairpin-like structures present themselves as streamwise velocity streaks and streamwise vorticity in the near-wall region. These structures influence 
TABLE III: Description of varying turbulent anisotropy states over the barycentric map (adapted from superquadratic glyphs generated by Jofre et al. ${ }^{58}$ ).

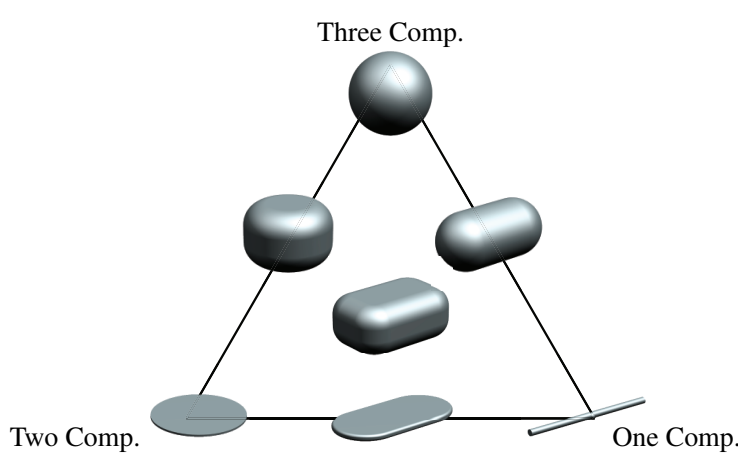

\begin{tabular}{lll}
\hline \hline Key & Turbulent State & Anisotropy Example \\
\hline One Comp. & $\overline{u^{\prime} u^{\prime}} \neq 0, \overline{v^{\prime} v^{\prime}}=\overline{w^{\prime} w^{\prime}}=0$ \\
Two Comp. & $\overline{u^{\prime} u^{\prime}}=\overline{v^{\prime} v^{\prime}} \neq 0, \overline{w^{\prime} w^{\prime}}=0$ \\
Three Comp. (Isotropic) & $\overline{u^{\prime} u^{\prime}}=\overline{v^{\prime} v^{\prime}}=\overline{w^{\prime} w^{\prime}}$ \\
Two Comp. Limit & $\overline{u^{\prime} u^{\prime}} \geq \overline{v^{\prime} v^{\prime}}, \overline{w^{\prime} w^{\prime}}=0$ \\
Axisymmetric Contraction & $\overline{u^{\prime} u^{\prime}}=\overline{v^{\prime} v^{\prime}} \geq \overline{w^{\prime} w^{\prime}}$ \\
Axisymmetric Expansion & $\overline{u^{\prime} u^{\prime}} \geq \overline{v^{\prime} v^{\prime}}=\overline{w^{\prime} w^{\prime}}$ \\
Other States & $\overline{u^{\prime} u^{\prime}} \neq \overline{v^{\prime} v^{\prime}} \neq \overline{w^{\prime} w^{\prime}}$ \\
\hline
\end{tabular}

the thermal field, where hot-streaks of fluid are localised to the streaks of low streamwise velocity and the local surface heat transfer is low.

\section{B. Turbulence Anisotropy}

The preceding subsection presented the good predictive capability of LES for complex turbulent flow regimes and their respective surface heat transfer. The flowfield predicted by the RANS $k-\omega$ SST simulation overpredicted reattachment length and Nusselt number distribution. The reattachment length and recirculation region are functions of the flow momentum and their predictions are dependant on the Reynolds stress field and their influence within the momentum transport equations. This section presents the turbulent anisotropy of the Reynolds stress field resolved by the LES. A comparison is then made to the Reynolds stresses calculated from the Boussinesq hypothesis, used in two-equation RANS model approaches, such as the $k$ - $\omega$ SST, and the EARSM model of Wallin and Johansson ${ }^{24}$ with near-wall treatment. The Reynolds stress field described by these closures are taken from the LiTT analysis allowing direct comparisons to those resolved by the LES.

Turbulence anisotropy represents the deviation in the Reynolds stress tensor to its isotropic state where $\overline{u^{\prime} u^{\prime}}=\overline{v^{\prime} v^{\prime}}=\overline{w^{\prime} w^{\prime}}=2 k / 3$. A tensor describing the turbulence anisotropy, $a_{i j}$, is calculated using Eq. 25 with the resolved LES Reynolds stresses and turbulent kinetic energy.

$$
a_{i j}=\frac{\overline{u_{i}^{\prime} u_{j}^{\prime}}}{k}-\frac{2}{3} \delta_{i j}
$$


Predictions of the turbulence anisotropy are made with the present LES data using Eq. 25. Visualisation of the anisotropy is achieved using Barycentric triangles. Unlike the classic invariance or Lumley maps, there is no visual bias to a particular turbulent state. The three corners represent the bounding behaviour of the anisotropy. One component turbulence presents turbulence with a single fluctuating component. Two component turbulence is a state at which two fluctuating components are present and are of equal value. Finally, three component turbulence represents an isotropic state. Each point within the triangle is a realizable state of the turbulence anisotropy. Table III presents a visual aid for the descriptions of turbulence where the dimensions of the shape reflect the relative magnitudes of the normal components. Formulation of the barycentric triangles is achieved by mapping the eigenvalues of $a_{i j}$ on to the triangle.

Figure 13 presents the barycentric triangles for the anisotropy resolved by the LES at six profiles across the recirculation and redeveloping regions. At the wall, all profiles express the twocomponent limit with near-equal components of streamwise and spanwise normal Reynolds stress. Away from the wall, the turbulence anisotropy progresses to the middle right section of the triangle. Through the recirculation region (Fig. 13a - 13b), the turbulence anisotropy progresses towards the axisymmetric contraction limit for the near-wall region $(Y / H<0.5)$. This condition represents the near-equal components of streamwise and spanwise normal Reynolds stress and an increasing normal component as the profile ascends from the wall. Following the departure from the axisymmetric limit, all profiles show the turbulence anisotropy develops towards the axisymmetric expansion limit prior to their descent back towards the two-component limit away from the wall. Within the redeveloping boundary layer, at $X / L_{r}=2.00$, the turbulence anisotropy, for a large section of the profile, is in close proximity to the plane-strain condition. The near-wall region is dictated by a similar curve to $X / L_{r}=1.50$, close to the two-component limit, but with a greater divergence from the axisymmetric contraction condition.

Clear deficits are present in the modelled anisotropy from the Boussinesq hypothesis. The formulation ensures the anisotropy is linear with the strain-rate, shown in Eq. 25, which is not true for most engineering flows. The time-averaged state of this flow regime is statistically twodimensional so $\overline{\partial / \partial z}=0$. It can then be shown that $\overline{w^{\prime} w^{\prime}}=\frac{2}{3} k$, while $\overline{u^{\prime} u^{\prime}}=\frac{2}{3} k-v_{t} \frac{\partial \bar{U}}{\partial x}$ and $\overline{v^{\prime} v^{\prime}}=\frac{2}{3} k+v_{t} \frac{\partial \bar{U}}{\partial x}$, when we consider the incompressible nature of this flow.

$$
a_{i j}^{B H}=-\frac{v_{t}}{k} S_{i j}
$$




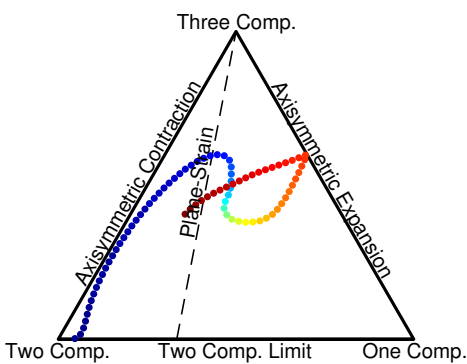

(a) $X / L_{r}=0.5$

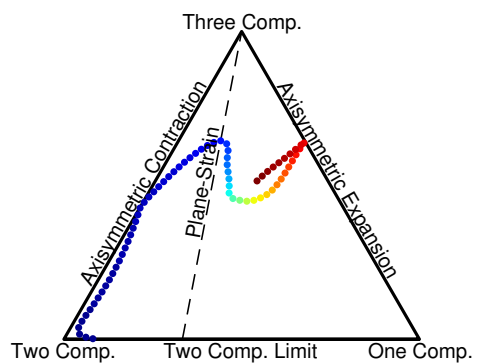

(b) $X / L_{r}=1.0$

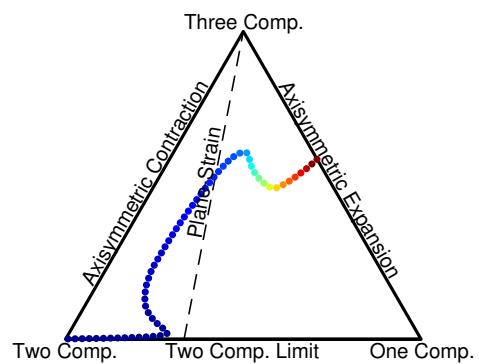

(c) $X / L_{r}=2.0$

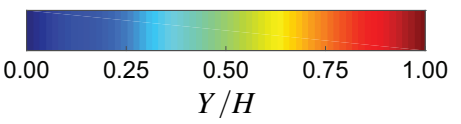

FIG. 13: Turbulent anisotropy resolved by the LES across the recirculation, reattachment and redevelopment regions. (a) $X / L_{r}=0.5$, (b) $X / L_{r}=1.0$ and (c) $X / L_{r}=2.0$.

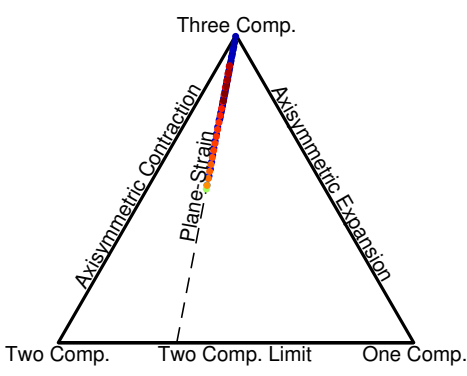

(a) $X / L_{r}=0.5$

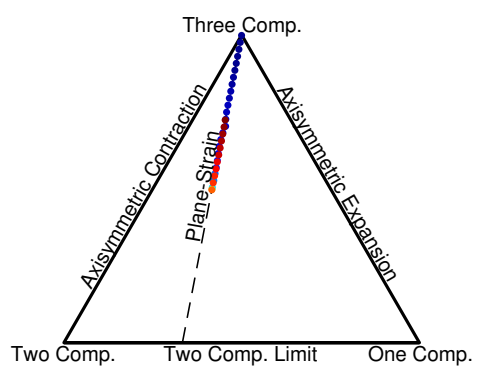

(b) $X / L_{r}=1.0$

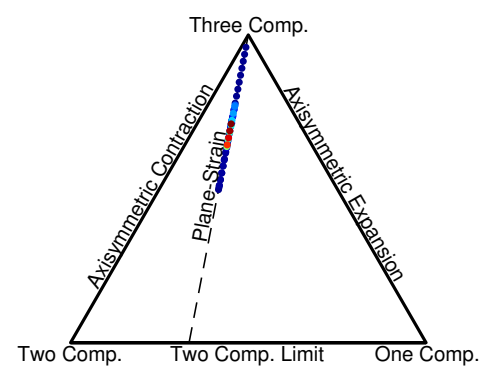

(c) $X / L_{r}=2.0$

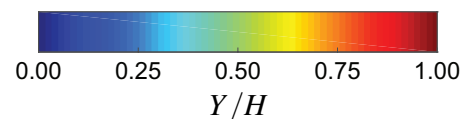

FIG. 14: Turbulence anisotropy of the Boussinesq hypothesis across the recirculation, reattachment and redevelopment regions. (a) $X / L_{r}=0.5$, (b) $X / L_{r}=1.0$ and (c) $X / L_{r}=2.0$.

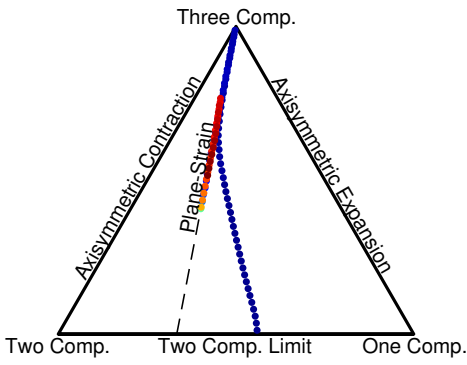

(a) $X / L_{r}=0.5$

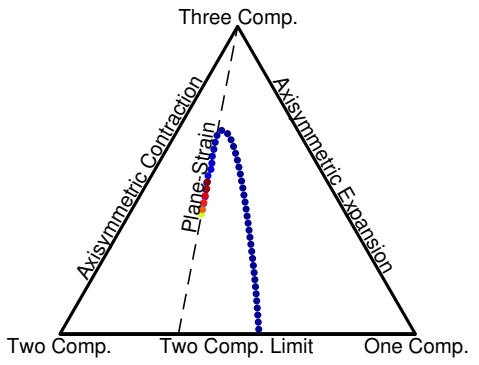

(b) $X / L_{r}=1.0$

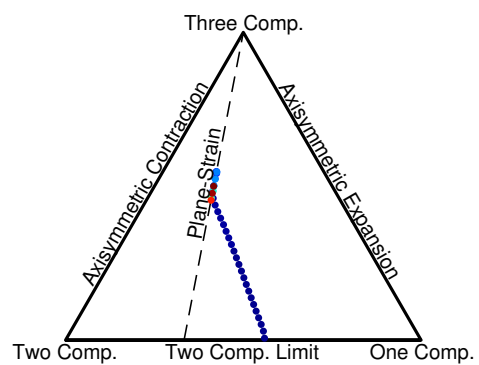

(c) $X / L_{r}=2.0$

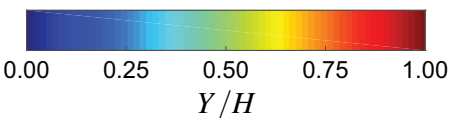

FIG. 15: Turbulence anisotropy of the EARSM across the recirculation, reattachment and redevelopment regions. (a) $X / L_{r}=0.5$, (b) $X / L_{r}=1.0$ and (c) $X / L_{r}=2.0$. 
Figure 14 presents the anisotropic state of the Reynolds stresses modelled from the Boussinesq hypothesis for a profile within the recirculation and redeveloping region. As expected, the plainstrain anisotropy is observed and comparisons to the resolved LES anisotropy of Figure 13 presents large differences in the anisotropic state. Alongside the plain-strain behaviour, the near-wall state of turbulence protrudes from an isotropic rather than a two-component state as seen by the wallrestricted normal fluctuations present in the LES solution.

The anisotropic state of the EARSM is shown in Figure 15. For two dimensional flows can also be analytically shown to follow this plane-strain state away from the wall, where $f_{1}=1$. However, the plain-strain behaviour is enhanced with the inclusion of the $\beta_{4}$ term, $S_{i k} \Omega_{k j}-\Omega_{i k} S_{k j}$. Like the Boussinesq hypothesis, the spanwise component $\overline{w^{\prime} w^{\prime}}=2 / 3 k$ and does not deviate from this state like the LES. The near-wall treatment enables a deviation for the plain-strain behaviour, which provides the two-component limit at the wall as shown in Figure 15. Although, the anisotropic behaviour is improved, deviation to the resolved LES is still observed.

Figures 16, 17 and 18 compare the Reynolds stress profiles of $\overline{u^{\prime} u^{\prime}}, \overline{v^{\prime} v^{\prime}}$ and $\overline{u^{\prime} v^{\prime}}$ respectively. Comparisons of the streamwise component, Figure 16, show the EARSM captures the peak values across the recirculation region where the turbulent fluctuations are enhanced. In the redeveloping boundary layer, the profiles remain in close agreement with those from the LES. Close to the wall, a small underprediction is shown following the expected peak value in turbulent boundary layer flows. Predictions of $\overline{u^{\prime} u^{\prime}}$ by the Boussinesq hypothesis show a considerable deviation for the LES, where the elevated stress in the recirculation region is underestimated. Figure 17 shows the normal Reynolds stress component, where the EARSM shows better agreement to the LES than the Boussinesq hypothesis. However, the rear of the recirculation region and downstream, the peak value occurs closer to the wall in the profile than shown by the LES. Comparisons of the turbulent shear stress, Figure 18, show that the Boussinesq hypthesis, with the LiTT description of eddy viscosity, is in very close agreement to the resolved LES stress. The EARSM predictions of $\overline{u^{\prime} v^{\prime}}$ repeat the behaviour shown in the normal stress component, $\overline{v^{\prime} v^{\prime}}$, where the peak location is present closer to the wall.

The impact of the different Reynolds stresses on the flow field can be qualitatively assessed by recalling the RANS momentum equation (Eq. 1). The smaller streamwise Reynolds stress component from the Boussinesq hypothesis would permit a greater streamwise momentum to balance the momentum transport equation. This would provide a longer recirculation region as is present in the full $k-\omega$ SST RANS simulation shown in Figure 7. An accurate prediction of the Reynolds 


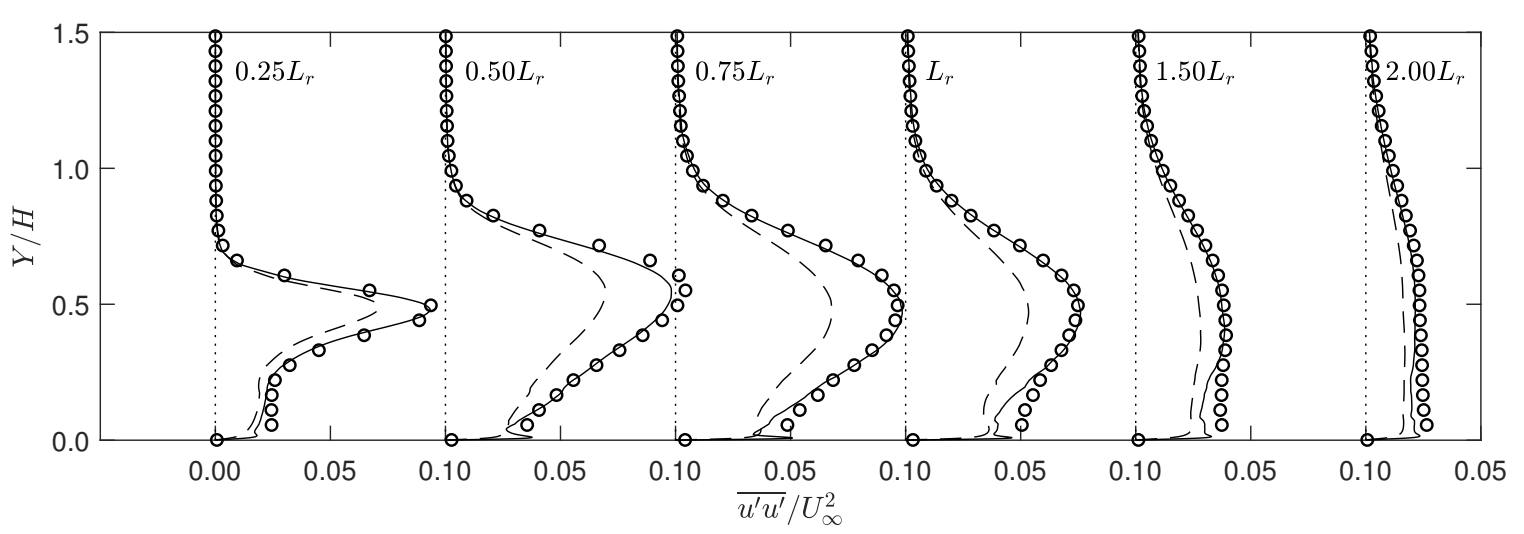

FIG. 16: Streamwise Reynolds stress $\left(\overline{u^{\prime} u^{\prime}}\right)$ comparison of constitutive relations to resolved LES. LES ( ०), LiTT-BH (-- ) and LiTT-EARSM (-).

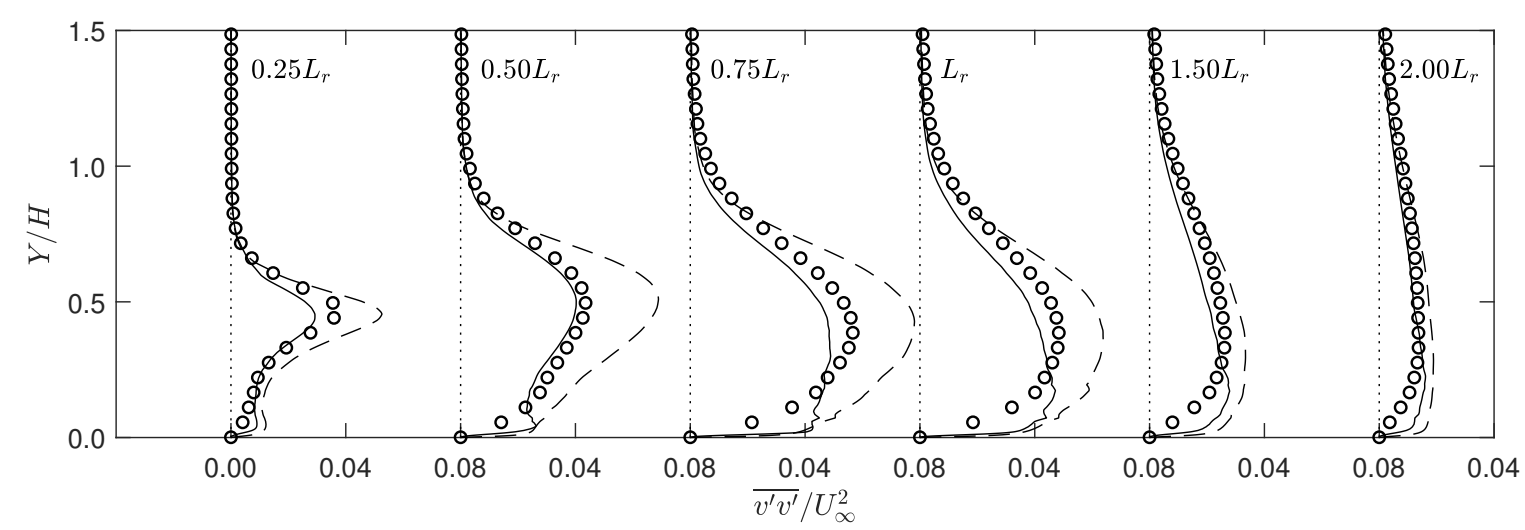

FIG. 17: Normal Reynolds stress $\left(\overline{v^{\prime} v^{\prime}}\right)$ comparison of constitutive relations to resolved LES.

Legend see Figure 16.

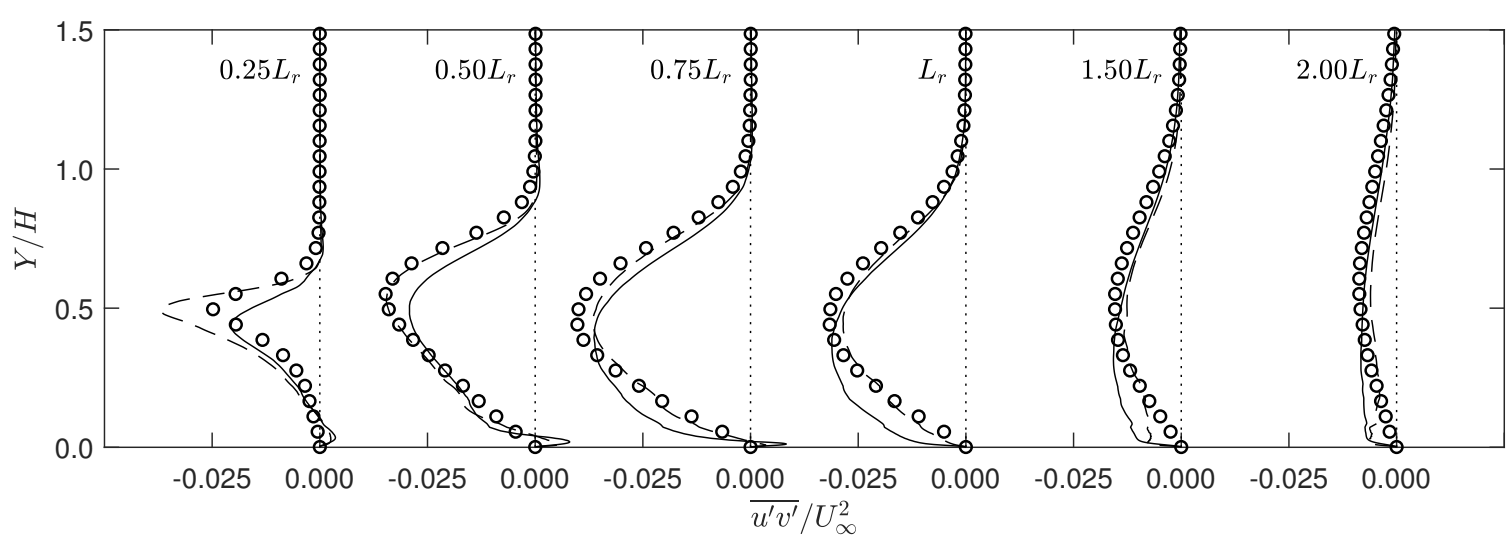

FIG. 18: Turbulent shear stress $\left(\overline{u^{\prime} v^{\prime}}\right)$ comparison of constitutive relations to resolved LES. Legend see Figure 16.

stress field, and the anisotropy in which the stresses are distributed to the momentum equations, 
is required to achieve a good estimation of the reattachment length and recirculation region. The EARSM shows the improvement and is a suitable candidate for separated and reattached flow regimes.

\section{Turbulent Heat Flux}

The previous section showed the complex turbulent anisotropy in this separated and reattached flow regime resolved by the LES. The LiTT analysis showed the Reynolds stress field provided by the Boussinesq hypothesis does not accurately capture the "true" anisotropy. This section investigates the accuracy of turbulent heat flux closures with the LiTT analysis, where the modelling of the turbulent heat flux is an important component of turbulent heat transfer predictions, alongside the Reynolds stresses.

LES predictions of turbulent heat flux are shown in Figure 19a and 19b for the streamwise $\left(\overline{u^{\prime} T^{\prime}}\right)$ and normal $\left(\overline{v^{\prime} T^{\prime}}\right)$ components respectively. Peak values are observed within the shear layer, where the increased presence of turbulent fluctuations enhance the mixing. The magnitude of the turbulent heat flux decreases in the downstream, redeveloping region, although the presence of the turbulent shear layer continues to be seen in downstream profiles.

TABLE IV: Table of coefficients for the studied diffusivity closures.

\begin{tabular}{cccc}
\hline \hline & Coefficient & Common Values & Value Used \\
\hline GDH & $P r_{t}$ & $0.8-1.0$ & 0.85 \\
GGDH & $c_{\theta}$ & 0.3 & 0.3 \\
HOGGDH & $c_{\theta}$ & $0.6^{29}$ & 0.4 \\
\hline \hline
\end{tabular}

Comparisons of the three RANS diffusion based closures for the turbulent heat flux vector, calculated from the LiTT analysis, are shown in the contours of Figure 19. Formulation of the Gradient Diffusion Hypothesis (GDH), Generalised GDH (GGDH) and Higher-Order GGDH (HOGGDH) are defined in Eq. 5, 6 and 7 respectively. The magnitude of the turbulent heat flux vector is controlled by the coefficients $\operatorname{Pr}_{t}$ and $c_{\theta}$ which are defined in Table IV. The HOGGDH closure, with the commonly used coefficient, achieved a large overprediction, and tests with a value of 0.4 showed a better agreement to those resolved by the LES. 

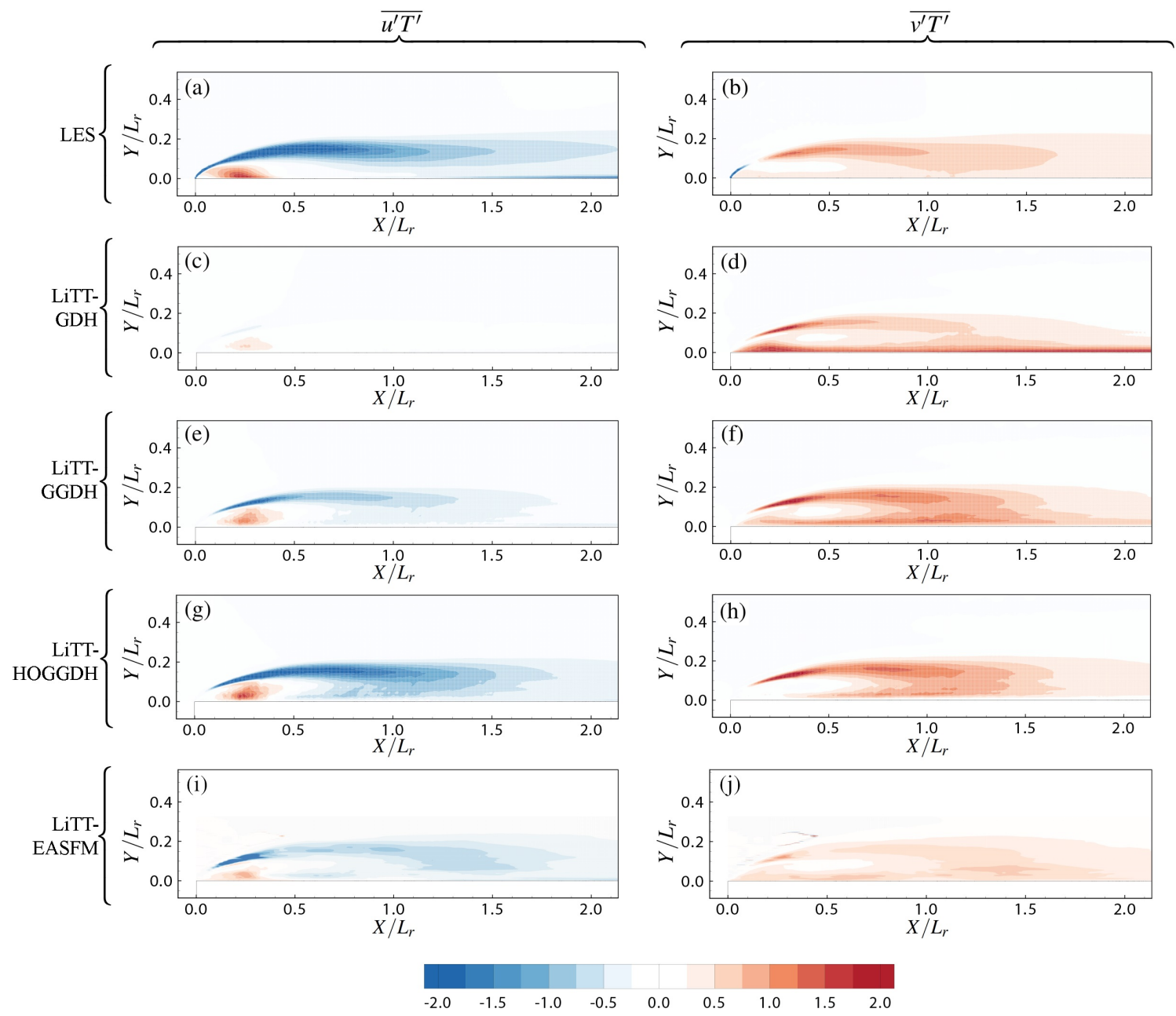

FIG. 19: Contours of streamwise and normal turbulent heat flux $\left(\overline{u^{\prime} T^{\prime}}\right.$ and $\overline{v^{\prime} T^{\prime}}$ respectively) for the LES ((a) $\overline{u^{\prime} T^{\prime}}$ and (b) $\overline{v^{\prime} T^{\prime}}$ ) and comparisons of GDH ((c) $\overline{u^{\prime} T^{\prime}}$ and (d) $\left.\overline{v^{\prime} T^{\prime}}\right)$, GGDH ((e) $\overline{u^{\prime} T^{\prime}}$ and (f) $\overline{v^{\prime} T^{\prime}}$ ), HOGGDH ((g) $\overline{u^{\prime} T^{\prime}}$ and (h) $\overline{v^{\prime} T^{\prime}}$ ) and EASFM ((i) $\overline{u^{\prime} T^{\prime}}$ and (j) $\overline{v^{\prime} T^{\prime}}$ ).

The closure of choice within most commercial CFD solvers is the GDH because of its simplicity. The GDH aligns the turbulent heat flux vector to the mean temperature gradient. Complex flows and their respective heat transfer do not comply to such a rule. A lack of streamwise temperature gradient within the current study results in a negligible streamwise turbulent heat flux component shown in Figure 19c. Figure 19d shows limited success in modelling the normal component, with good agreement seen in the shear layer, but an overprediction in the near-wall region where high normal gradients are present from the prescribed wall heat flux.

Inclusion of the Reynolds stresses in the GGDH and HOGGDH closures provide a degree of anisotropy to the turbulent diffusivity, permitting the turbulent heat flux to deviate from the alignment to the mean temperature gradient provided by the GDH closure. The quadratic products 


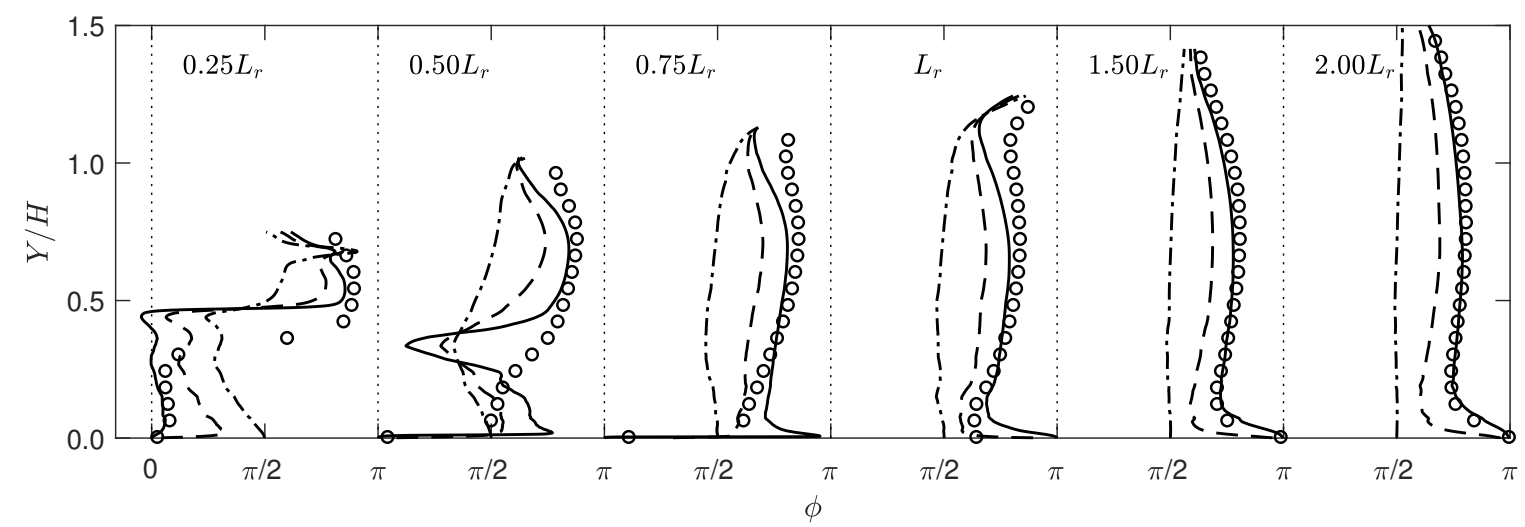

FIG. 20: Comparison of the angle of the turbulent heat flux vector, $\phi$, between the resolved LES and RANS diffusion hypothesis closures. LES ( ० ), LiTT-GDH (-- ), LiTT-GGDH (---) and LiTT-HOGGDH (-).

of the Reynolds stresses in the HOGGDH shows qualitatively better agreement to the turbulent heat flux contours of the LES. Deviations to the magnitude are present and some overprediction exists in the near-wall region. The results of the EASFM predictions of turbulent heat flux are shown in Figure 19i and 19j for the streamwise and normal components respectively. The two contours show an underprediction across the flow when compared to the LES, although the general trends are captured, showing the elevated turbulent heat flux across the shear layer and downstream.

A useful measure for assessing the suitability of these closure is the angle formed by the modelled turbulent heat flux. The angle, $\phi$, is defined by the normal and streamwise turbulent heat flux (Eq. 27).

$$
\overline{\overline{u^{\prime} T^{\prime}}}=\tan \phi
$$

Figure 20 presents the turbulent heat flux angles from four profiles across the recirculation and redeveloping region. Within the redeveloping region, the modelled angle described by the HOGGDH closure is in close agreement with the turbulent heat flux resolved by the LES. Angles defined by the GDH and GGDH do not agree with the LES, providing a greater contribution to the normal component. In the near-wall region, a angle difference of $\pi / 2$ is present between the LES and the GDH, highlighting the defects of the standard GDH model's ability to provide accurate turbulent diffusion close to the wall. Within the recirculation region $\left(0.5 L_{r}\right)$, large differences are observed between the angles of the LES results and all closures. The LiTT analysis shows that the 


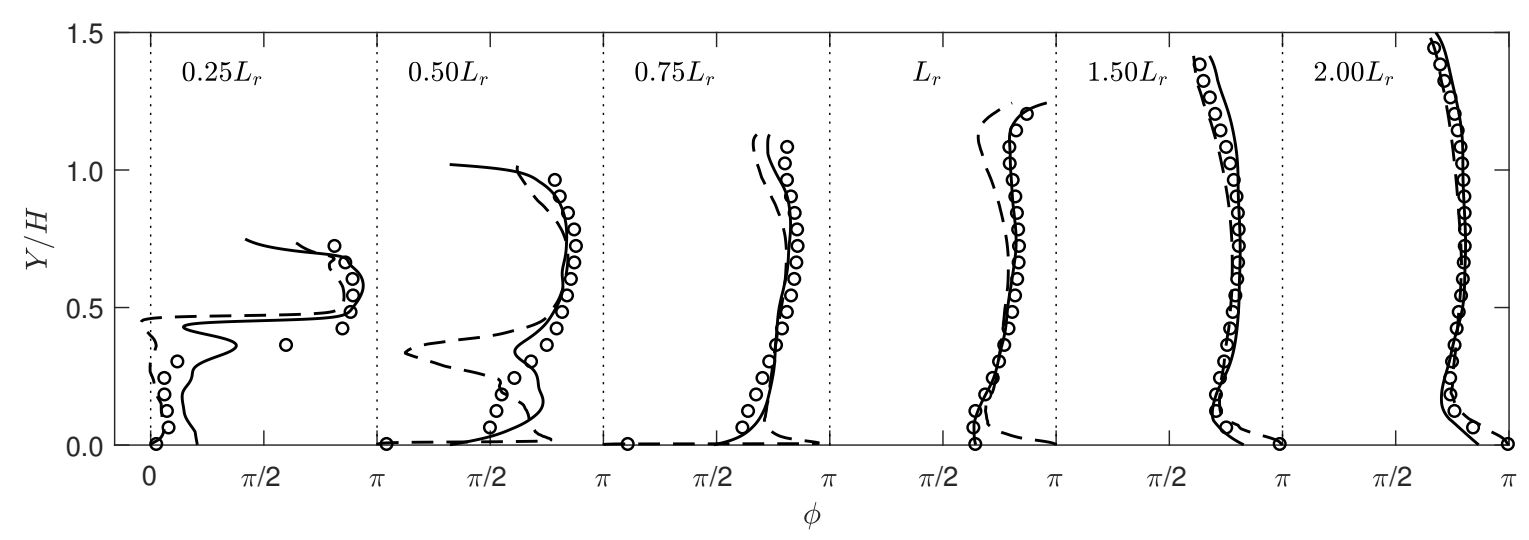

FIG. 21: Comparison of the angle of the turbulent heat flux vector, $\phi$, between the resolved LES, HOGGDH and EASFM models. LES ( $\circ$ ), LiTT-HOGGDH (- - -) and LiTT-EASFM (-).

HOGGDH prediction of the turbulent heat flux angle better describes the turbulent heat flux than the GDH and GGDH closures.

Figure 21 shows the turbulent heat flux angle predicted by the EASFM and compared against the HOGGDH and LES results. The EASFM results show the angle is in better agreement to the LES than the HOGGDH over all profiles. At $0.5 L_{r}$, the EASFM show a small deviation from the LES, but is in better agreement with the profile than the diffusion hypothesis closures. Within the reattachment location and redeveloping regions, the EASFM shows agreement across the full profile where the HOGGDH showed some deviation away from the wall. However, the turbulent heat flux magnitude predicted by the EASFM is underestimated.

With good agreement of the turbulent heat flux shown by the HOGGDH model in Fig. 19 and Fig. 20, an optimal spatial prediction of it's coefficient, $c_{\theta}$, is sought to achieve the correct magnitude of the turbulent heat flux vector. Calibrating the coefficient by a single component of the turbulent heat flux can provide conflicting values (as shown in Figure 22) for regions where $\phi$ is not accurately captured. This is true within the recirculation region where the angle dictated by the model is shown to deviate from the LES prediction. An optimal coefficient is derived using a weighted approach described by Eq. 28. Milani et al. ${ }^{59}$ used such an approach for calculating an LES isotropic turbulent diffusivity field to improve GDH predictions. The optimal coefficient exists between a calibration based upon $\overline{u^{\prime} T^{\prime}}$ and $\overline{v^{\prime} T^{\prime}}$. Figure 22 shows that over the shear layer, the optimal coefficient has a peak within the recirculation region followed by a minima at the reattachment location before ascending across the redeveloping region. Over the initial shear layer development a large, optimal $c_{\theta}$, attempts to obtain the large turbulent heat flux values that are present in the LES turbulent heat flux. 


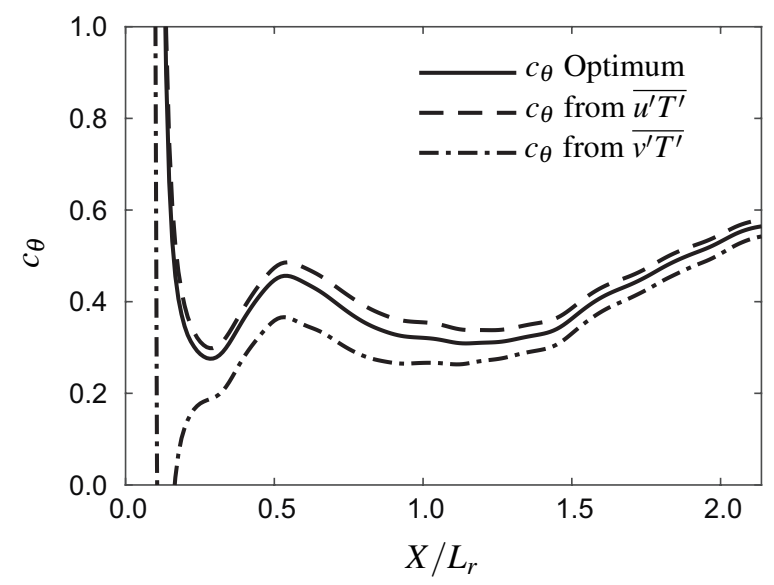

FIG. 22: An optimal value of $c_{\theta}$ through the shear layer and downstream with comparison to directionally calibrated coefficients.

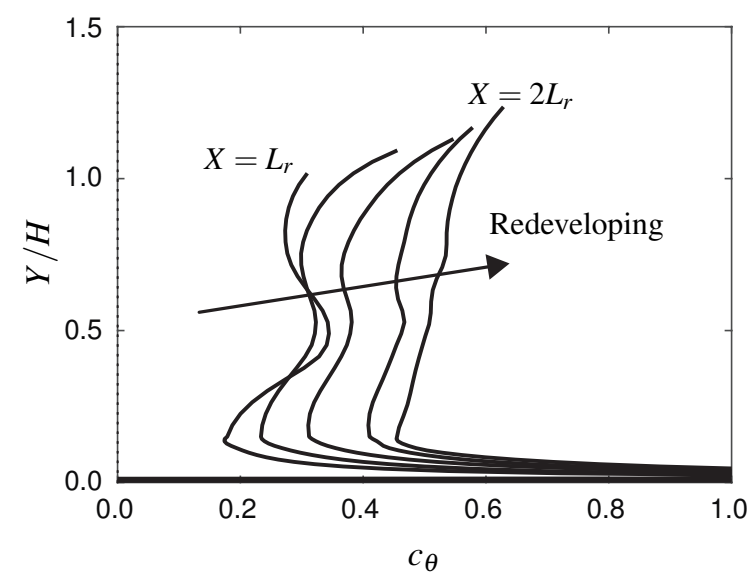

FIG. 23: Profiles of optimal $c_{\theta}$ across the redeveloping region.

$$
c_{\theta, o p t}=-\frac{k}{\tau_{t}} \frac{\overline{u_{i}^{\prime} T^{\prime}}\left(\overline{u_{i}^{\prime} u_{k}^{\prime}} \overline{u_{k}^{\prime} u_{j}^{\prime}} \frac{\partial \bar{T}}{\partial x_{j}}\right)}{\left(\overline{u_{l}^{\prime} u_{m}^{\prime}} \overline{u_{m}^{\prime} u_{n}^{\prime}} \frac{\partial \bar{T}}{\partial x_{n}}\right)\left(\overline{u_{l}^{\prime} u_{m}^{\prime}} \overline{u_{m}^{\prime} u_{n}^{\prime}} \frac{\partial \bar{T}}{\partial x_{n}}\right)}
$$

The increasing optimal $c_{\theta}$ across the redeveloping region, seen in Figure 22, is investigated further through profiles presented in Figure 23. Profiles are equally spaced between $X=L_{r}$ and $X=2 L_{r}$. Values are blanked where the turbulent heat flux vector is small $\left(\left|\overline{u_{i}^{\prime} T^{\prime}}\right|<0.1\right)$. Away from the wall, a peak $c_{\theta}$ is shown within the profile, at a location consistent with the shear layer location. The peak is observed to decay downstream where the profile tends towards a linear distribution. As the redevelopment of the boundary layer progresses, the optimal $c_{\theta}$ is shown to increase but does not tend towards an asymptotic value at this early stage of redevelopment. Within the nearwall region, a large value of $c_{\theta}$ is required across all profiles where a considerable streamwise 
component is present. The results highlight that a constant $c_{\theta}$ will evidently show deviations, but a range of plausible constants exist from this a priori analysis covering the differing regions of this flow. Modelling improvements could be furthered by introduction of a spatially varying $c_{\theta}$ following the results shown.

The investigation of closures and the derivation of an optimal spatial $c_{\theta}$ has been made by use of the Reynolds stresses resolved by the LES. However, common two-equation RANS models will construct the Reynolds stress field from the Boussinesq hypothesis or at best use an EARSM approach as shown earlier. As Reynolds stresses predicted by the Boussinesq hypothesis deviate from those resolved by the LES, the angle and magnitude of the turbulent heat flux vector will deviate from the LiTT analysis. Reynolds stresses modelled by the EARSM were shown to be closely aligned to those predicted by the LES and will provide an improved Reynolds stress field for the HOGGDH closure

\section{CONCLUSIONS}

LES of the geometrically simple heated blunt plate showed a variety of complex turbulent behaviours for the moderate Reynolds number of 21,600 studied. Such a configuration holds physical relevance to many engineering flow problems. This paper showed that LES using the WALE sub-grid scale model provided accurate predictions of the surface heat transfer and turbulent flow behaviour. An LES informed Turbulent Transport (LiTT) analysis allowed for closures used in RANS to be directly compared to the well-resolved LES flow field and the main findings of the comparison can be summarised below.

- LES results showed the near-wall anisotropy is characterised by a profile ascending from the two-component limit. Within the recirculation bubble the profiles develop towards the turbulence axisymmetric contraction limit. In the redeveloping region, the turbulence anisotropy develops to a state closer to the plane-strain behaviour exhibited by the Reynolds stresses modelled with the Boussinesq hypothesis and the EARSM away from the wall.

- Results of the EARSM show good agreement to the streamwise and normal component of the Reynolds stress tensor. The anisotropic state with near-wall treatment provides the trend from the two-component limit is provided, but outside of this treatment, the flow still remains in a plane-strain anisotropic state. 
- The GDH and GGDH closures fail to reproduce the turbulent heat flux vector. The HOGGDH successfully improves predictions of the turbulent heat flux angle in the redeveloping region but deviates in the complex recirculating flow prior to the reattachment location. The complex EASFM model improved the modelled angle across all regions of the flow, but did not agree with the magnitude shown in the LES.

- A spatially varying coefficient, $c_{\theta}$, for the HOGGDH turbulent heat flux closure was produced to achieve the magnitude of the LES resolved turbulent heat flux and show ideal values for advancing modelling knowledge. A large value is required in the near-wall region to provide the correct magnitude while the redeveloping region aft of the recirculation region showed values ranging from 0.3 to 0.6 . Contours showed the turbulent heat flux field was comparable to the LES.

This paper used well-resolved LES data to demonstrate that the common closures used in RANS simulations do not accurately capture the Reynolds stress and turbulent heat flux fields in turbulent separated and reattached flow. Existing models such as EARSM, HOGGDH and EASFM models show improvements but do not provide a robust solution yet. Our work highlights the need for an advanced turbulent heat flux model that will accommodate improvements to the modelled turbulent heat flux vector within the shear layer and recirculation region, where the turbulent heat flux angle is well predicted by the EASFM but the magnitude is underpredicted. Recent advances to data-driven modelling using high-fidelity datasets demonstrates itself as an encouraging resolution to closure problems of which the current study provides a foundation.

\section{NOMENCLATURE}

\section{Roman and Greek}

$a_{i j} \quad$ Turbulence anisotropy tensor

$\begin{array}{ll}c_{\theta} & \text { Model coefficient }\end{array}$

$C_{f} \quad$ Skin friction coefficient

$H \quad$ Plate Thickness $(m)$

$k \quad$ Turbulent kinetic energy $\left(\mathrm{m}^{2} / \mathrm{s}^{2}\right)$ or Thermal conductivity $(\mathrm{W} / \mathrm{mK})$

$L_{r} \quad$ Reattachment length $(m)$

p Pressure $(\mathrm{Pa})$ 


$\begin{array}{ll}\overline{u_{i}^{\prime} u_{j}^{\prime}} & \text { Reynolds stress tensor }\left(\mathrm{m}^{2} / \mathrm{s}^{2}\right) \\ \overline{u_{i}^{\prime} T^{\prime}} & \text { Turbulent heat flux vector }(\mathrm{m} K / \mathrm{s}) \\ S_{i j} & \text { Strain rate tensor }\left(\mathrm{s}^{-1}\right) \\ T & \text { Temperature }(\mathrm{K}) \\ U, V, W & \text { Velocity }(\mathrm{m} / \mathrm{s}) \\ u^{\prime}, v^{\prime}, w^{\prime} & \text { Fluctuating velocity }(\mathrm{m} / \mathrm{s}) \\ \alpha_{t} & \text { Turbulent diffusivity }\left(\mathrm{m}^{2} / \mathrm{s}\right) \\ \delta_{i j} & \text { Kronecker delta } \\ v_{t} & \text { Eddy/Turbulent viscosity }\left(\mathrm{m}^{2} / \mathrm{s}\right) \\ \phi & \text { Turbulent heat flux angle } \\ \rho & \text { Density }\left(\mathrm{kg} / \mathrm{m}^{3}\right) \\ \tau_{t} & \text { Turbulent time scale }(\mathrm{s}) \\ \omega & \text { Specific dissipation rate }(1 / \mathrm{s})\end{array}$

\section{Subscript}

$\infty$

Freestream parameter

res

Resolved parameter

rms

Root-mean-square parameter

sgs

Sub-grid scale parameter

w

Wall value

\section{Non-dimensional Numbers}

$\mathrm{Nu} \quad$ Nusselt number

$P r_{t} \quad$ Turbulent Prandtl number

Re Reynolds number

Acronyms

EARSM Explicit Algebraic Reynolds Stress Model

EASFM Explicit Algebraic Scalar Flux Model

GDH Gradient Diffusion Hypothesis

GGDH Generalised Gradient Diffusion Hypothesis

HOGGDH Higher-Order Generalised Gradient Diffusion Hypothesis

LES Large Eddy Simulation

LiTT LES informed Turbulent Transport 


$\begin{array}{ll}\text { PDA } & \text { Pulse Dopler Anemometry } \\ \text { RANS } & \text { Reynolds-Averaged Navier Stokes } \\ \text { SGS } & \text { Sub-Grid Scale } \\ \text { WALE } & \text { Wall-Adaptive Local Eddy-viscosity }\end{array}$

\section{ACKNOWLEDGMENTS}

The authors would like to acknowledge the use of Athena at HPC Midlands+, which was funded by the EPSRC on grant EP/P020232/1. This research was supported by EPSRC (Engineering and Physical Sciences Research Council) Centre for Doctoral Training in Gas Turbine Aerodynamics EP/L015943/1.

\section{REFERENCES}

${ }^{1}$ T. Ota and N. Kon, "Heat transfer in the separated and reattached flow on a blunt flat plate," J. Heat Transf. 96, 459-462 (1974).

${ }^{2}$ T. Ota and H. Nishiyama, "A correlation of maximum turbulent heat transfer coefficient in reattachment flow region,” Int. J. Heat Mass Tran. 30, 1193-1200 (1987).

${ }^{3}$ T. Ota and N. Kon, "Heat transfer in the separated and reattached flow over blunt flat plates ‘ $\breve{A}$ ‘Ťeffects of nose shape,’ Int. J. Heat Mass Tran. 22, 197-206 (1979).

${ }^{4}$ T. Ota and N. Kon, "Turbulent transfer of momentum and heat in a separated and reattached flow over a blunt flat plate," J. Heat Transf. 102, 749-754 (1980).

${ }^{5}$ H. Nishiyama, T. Ota, and K. Sato, "Temperature fluctuations in a separated and reattached turbulent flow over a blunt flat plate," Warme- und Stoffubertragung 23, 275-281 (1988).

${ }^{6}$ T. Ota and M. Narita, "Turbulence measurements in a separated and reattached flow over a blunt flat plate," J. Fluids Eng. 100, 224-228 (1978).

${ }^{7}$ N. Djilali and I. S. Gartshore, "Turbulent flow around a bluff rectangular plate. Part I: Experimental investigation,” J. Fluids Eng. 113, 51-59 (1991).

${ }^{8}$ M. Kiya and K. Sasaki, “Structure of turbulent separation bubble," J. Fluid Mech. 137, 83-113 (1983).

${ }^{9}$ M. Kiya and K. Sasaki, "Structure of large-scale vortices and unsteady reverse flow in the reattaching zone of a turbulent separation bubble," J. Fluid Mech. 154, 463-491 (1985). 
${ }^{10}$ T. Ota and H. Yanaoka, "Numerical analysis of a separated and reattached flow over a blunt flat plate,” in 5th Int'l Symp. CFD, Vol. 2 (Jpn. Soc. CFD, Sendai, 1993) pp. 423-428.

${ }^{11}$ D. K. Tafti and S. P. Vanka, “A numerical study of flow separation and reattachment on a blunt plate,” Phys. Fluids A-Fluid 3, 1749-1759 (1991).

${ }^{12}$ H. Yanaoka, H. Yoshikawa, and T. Ota, "Direct numerical simulation of turbulent separated flow and heat transfer over a blunt flat plate," J. Heat Transf. 125, 779-787 (2003).

${ }^{13}$ K. Sugawara, H. Yoshikawa, and T. Ota, "LES of turbulent separated flow and heat transfer in a symmetric expansion plane channel,” J. Fluid Eng. 127, 865 (2005).

${ }^{14}$ Z. Yang and I. E. Abdalla, "On secondary instability of a transitional separation bubble," Comput. Fluids 179, 595-603 (2019).

${ }^{15} \mathrm{M}$. Langari and Z. Yang, "Numerical study of the primary instability in a separated boundary layer transition under elevated free-stream turbulence,” Phys. Fluids 25, 074106 (2013).

${ }^{16} \mathrm{H}$. J. Li and Z. Yang, "Separated boundary layer transition under pressure gradient in the presence of free-stream turbulence," Phys. Fluids 31, 104106 (2019).

${ }^{17}$ A. Cimarelli, A. Leonforte, E. De Angelis, A. Crivellini, and D. Angeli, "Resolved dynamics and subgrid stresses in separating and reattaching flows," Phys. Fluids 31, 095101 (2019).

${ }^{18}$ R. Pain, P.-E. Weiss, S. Deck, and J.-C. Robinet, "Large scale dynamics of a high reynolds number axisymmetric separating/reattaching flow," Phys. Fluids 31, 125119 (2019).

${ }^{19}$ X. Chen and H. Xia, "Hybrid les-rans study of an effusion cooling array with circular holes," Int. J. Heat Fluid Fl. 77, 171-185 (2019).

${ }^{20} \mathrm{H}$. Xia, "Turbulent jet characteristics for axisymmetric and serrated nozzles," Comput. Fluids 110, 189-197 (2015).

${ }^{21}$ J. Boussinesq, Essai sur la Théorie des eaux Courantes (Impr. Nationale, 1877).

${ }^{22}$ C. Speziale, "Analytical methods for the development of Reynolds-stress closures in turbulence," Annu. Rev. Fluid Mech. 23, 107-157 (1991).

${ }^{23}$ S. B. Pope, “A more general effective-viscosity hypothesis," J. Fluid Mech. 72, 331-340 (1975).

${ }^{24}$ S. Wallin and A. V. Johansson, "An explicit algebraic reynolds stress model for incompressible and compressible turbulent flows," J. Fluid Mech. 403, 89-132 (2000).

${ }^{25}$ S. E. Elghobashi and T. W. Abou-Arab, "A two-equation turbulence model for two-phase flows," Phys. Fluids 26, 931-938 (1983).

${ }^{26}$ W. M. Kays, “Turbulent Prandtl Number-Where are We?” J. Heat Transf. 116, 284-295 (1994). 
${ }^{27}$ D. Lakehal, "Near-wall modeling of turbulent convective heat transport in film cooling of turbine blades with the aid of direct numerical simulation data," J. Turbomach. 124, 485 (2002).

${ }^{28}$ B. J. Daly and F. H. Harlow, “Transport equations in turbulence," Phys. Fluids 13, 2634-2649 (1970).

${ }^{29} \mathrm{~K}$. Abe and K. Suga, "Towards the development of a reynolds-averaged algebraic turbulent scalar-flux model,” Int. J. Heat Fluid Fl. 22, 19-29 (2001).

${ }^{30}$ C. Le Ribault and R. Friedrich, "Investigation of Transport Equations for Turbulent Heat Fluxes in Compressible Flows,” Int. J. Heat Mass Tran. 40, 2721-2738 (1997).

${ }^{31}$ J. Ling, K. J. Ryan, J. Bodart, and J. K. Eaton, “Analysis of Turbulent Scalar Flux Models for a Discrete Hole Film Cooling Flow,” J. Turbomach. 138 (2015).

${ }^{32} \mathrm{P}$. Wikström, S. Wallin, and A. V. Johansson, "Derivation and investigation of a new explicit algebraic model for the passive scalar flux," Phys. Fluids 12, 688-702 (2000).

${ }^{33}$ The OpenFOAM Foundation, “OpenFOAM v5.0," https://openfoam.org/ (2011-2019).

${ }^{34}$ F. Gori, M. Angelino, A. Boghi, and I. Petracci, "Preliminary numerical solutions of the evolution of free jets," in ASME 2012 Int. Mech. Eng. Cong. and Expo. (ASME Digital Collection, 2012) pp. 463-469.

${ }^{35} \mathrm{M}$. Angelino, A. Boghi, and F. Gori, "Numerical solution of three-dimensional rectangular submerged jets with the evidence of the undisturbed region of flow," Numer. Heat Tr. A-Appl 70, 815-830 (2016).

${ }^{36}$ K. Yamamoto, T. Murota, T. Okazaki, and M. Taniguchi, "Large eddy simulation of a pulverized coal jet flame ignited by a preheated gas flow," P. Combust. Inst. 33, 1771-1778 (2011).

${ }^{37}$ M. Dianat, M. Skarysz, and A. Garmory, "A coupled level set and volume of fluid method for automotive exterior water management applications," Int. J. Multiphas. Flow 91, 19 - 38 (2017).

${ }^{38}$ F. Nicoud and F. Ducros, "Subgrid-scale stress modelling based on the square of the velocity gradient tensor," Flow Turbul. Combust. 62, 183-200 (1999).

${ }^{39}$ F. Duchaine, A. Corpron, L. Pons, V. Moureau, F. Nicoud, and T. Poinsot, "Development and assessment of a coupled strategy for conjugate heat transfer with large eddy simulation: Application to a cooled turbine blade," Int. J. Heat Fluid Fl. 30, 1129-1141 (2009).

${ }^{40}$ F. Duchaine, M. Boileau, Y. Sommerer, and T. Poinsot, "Large eddy simulation of flow and heat transfer around two square cylinders in a tandem arrangement," J. Heat Transf. 136, 101702 (2014). 
${ }^{41}$ S. Scholl, T. Verstraete, F. Duchaine, and L. Gicquel, "Conjugate heat transfer of a ribroughened internal turbine blade cooling channel using large eddy simulation,” Int. J. Heat Fluid Fl. 61, 650-664 (2016).

${ }^{42}$ S. Qin, M. Koochesfahani, and F. Jaberi, "Large eddy simulations of unsteady flows over a stationary airfoil," Comput. Fluids 161, 155-170 (2018).

${ }^{43}$ M. Draksler, B. Končar, and L. Cizelj, "On the accuracy of large eddy simulation of multiple impinging jets,” Int. J. Heat Mass Tran. 133, 596 - 605 (2019).

${ }^{44}$ M. Kim, J. Lim, S. Kim, S. Jee, J. Park, and D. Park, "Computations of turbulent transition in boundary layer with les," in AIAA Aviation 2019 Forum (2019) p. 2838.

${ }^{45}$ A. Schindler, B. A. Younis, and B. Weigand, "Large-eddy simulations of turbulent flow through a heated square duct," Int. J. Therm. Sci. 135, 302-318 (2019).

${ }^{46}$ T. M. Eidson, "Numerical Simulation of the Turbulent Rayleigh-Benard Problem using Subgrid Modeling,” J. Fluid Mech. 158, 245-268 (1985).

${ }^{47}$ G. Grötzbach and M. Wörner, "Direct Numerical and Large Eddy Simulations in Nuclear Applications," Int. J. Heat Fluid Fl. 20, 222-240 (1999).

${ }^{48}$ A. Salvagni, D. Borello, F. Rispoli, and K. Hanjalić, "Large-Eddy Simulations of Heat Transfer in Asymmetric Rib-roughened Ducts: Effects of Rotation,” Int. J. Heat Fluid Fl. 68, 373-385 (2017).

${ }^{49}$ J. Pallares and L. Davidson, "Large-eddy simulations of turbulent heat transfer in stationary and rotating square ducts," Phys. Fluids 14, 2804-2816 (2002).

${ }^{50} \mathrm{H}$. Raiesi, U. Piomelli, and A. Pollard, "Evaluation of turbulence models using direct numerical and large-eddy simulation data," J. Fluids Eng. 133 (2011).

${ }^{51}$ S. Parneix, D. Laurence, and P. A. Durbin, “A procedure for using DNS databases," J. Fluids Eng. 120, 40-47 (1998).

${ }^{52}$ P. A. Durbin, "Some recent developments in turbulence closure modeling," Annu. Rev. Fluid Mech. 50, 77-103 (2018).

${ }^{53}$ J. Weatheritt and R. D. Sandberg, "The development of algebraic stress models using a novel evolutionary algorithm,” Int. J. Heat Fluid Fl. 68 (2017).

${ }^{54} \mathrm{~F}$. Menter and T. Esch, "Elements of industrial heat transfer predictions," in 16th Brazilian Cong. Mech. Eng. (COBEM), Vol. 109 (2001) p. 650.

${ }^{55}$ F. R. Menter, M. Kuntz, and R. Langtry, "Ten years of industrial experience with the SST turbulence model," Turb. Heat Mass Trans. 4, 625-632 (2003). 
${ }^{56}$ T. Ota, Y. Asano, and J. Okawa, "Reattachment Length and Transition of the Separated Flow over Blunt Flat Plates," Bulletin of the JSME 24 (1981).

${ }^{57}$ E. M. Sparrow, S. S. Kang, and W. Chuck, "Relation between the points of flow reattachment and maximum heat transfer for regions of flow separation," Int. J. Heat Mass Trans. 30, 12371246 (1987).

${ }^{58}$ L. Jofre, S. P. Domino, and G. Iaccarino, “A framework for characterizing structural uncertainty in large-eddy simulation closures," Flow Turbul. Combust. 100, 341-363 (2018).

${ }^{59}$ P. M. Milani, J. Ling, G. Saez-Mischlich, J. Bodart, and J. K. Eaton, “A machine learning approach for determining the turbulent diffusivity in film cooling flows," J. Turbomach 140 (2017). 


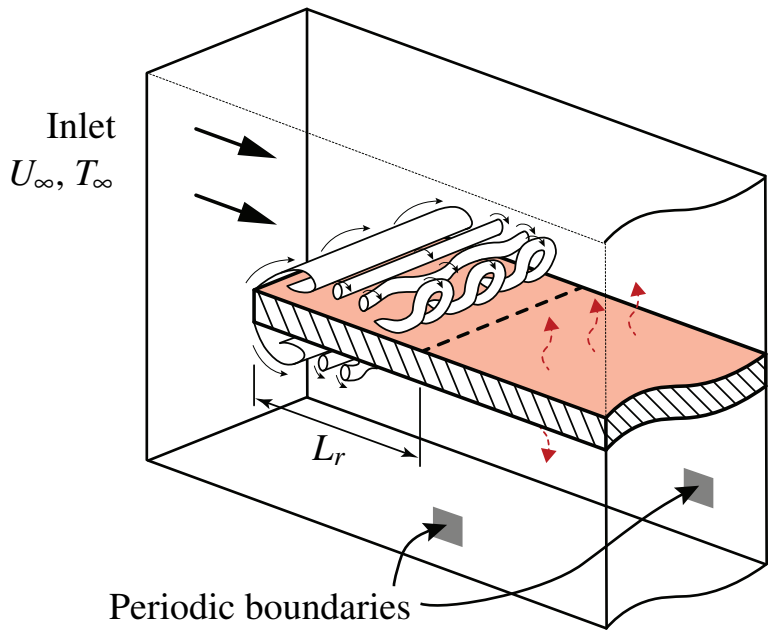




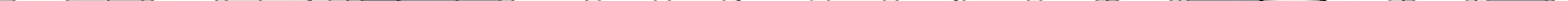




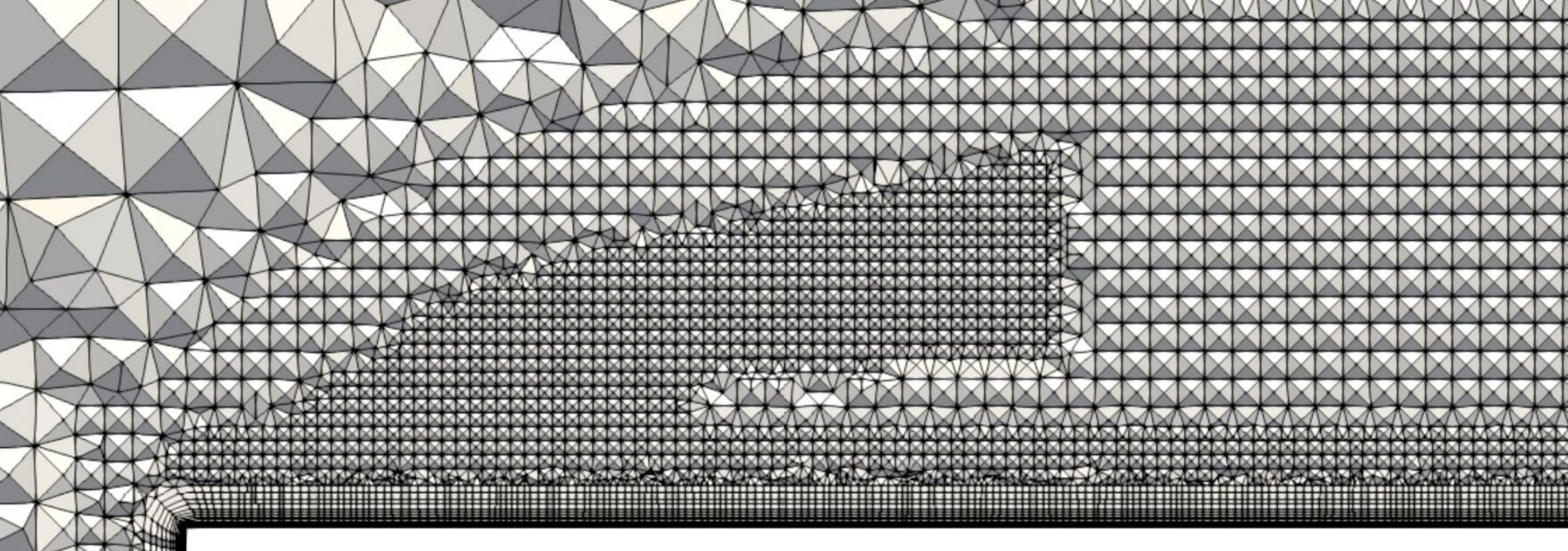


(b)

(c)

(a) Slice $Z=0$

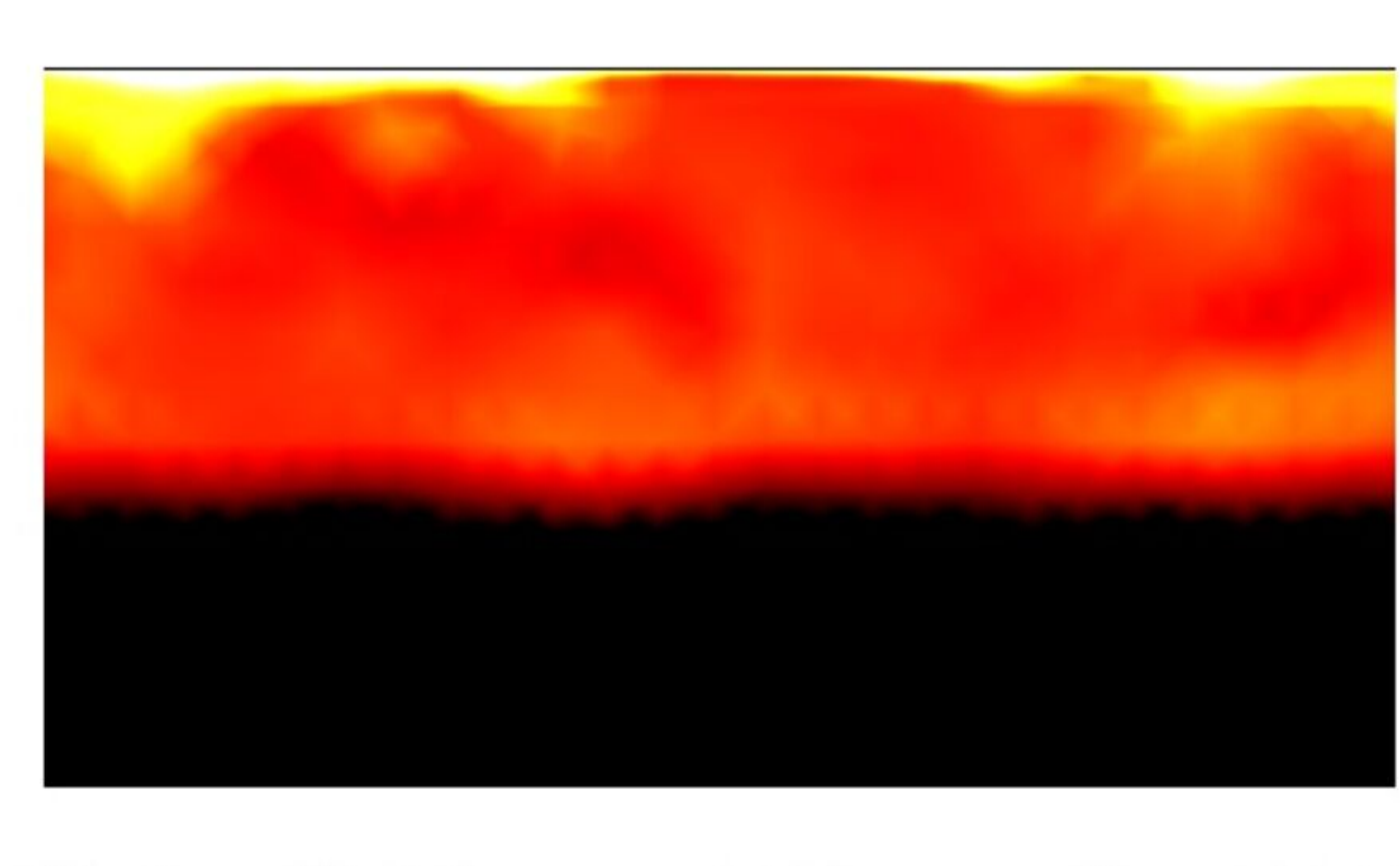

(b) Slice $X=2.5 H$

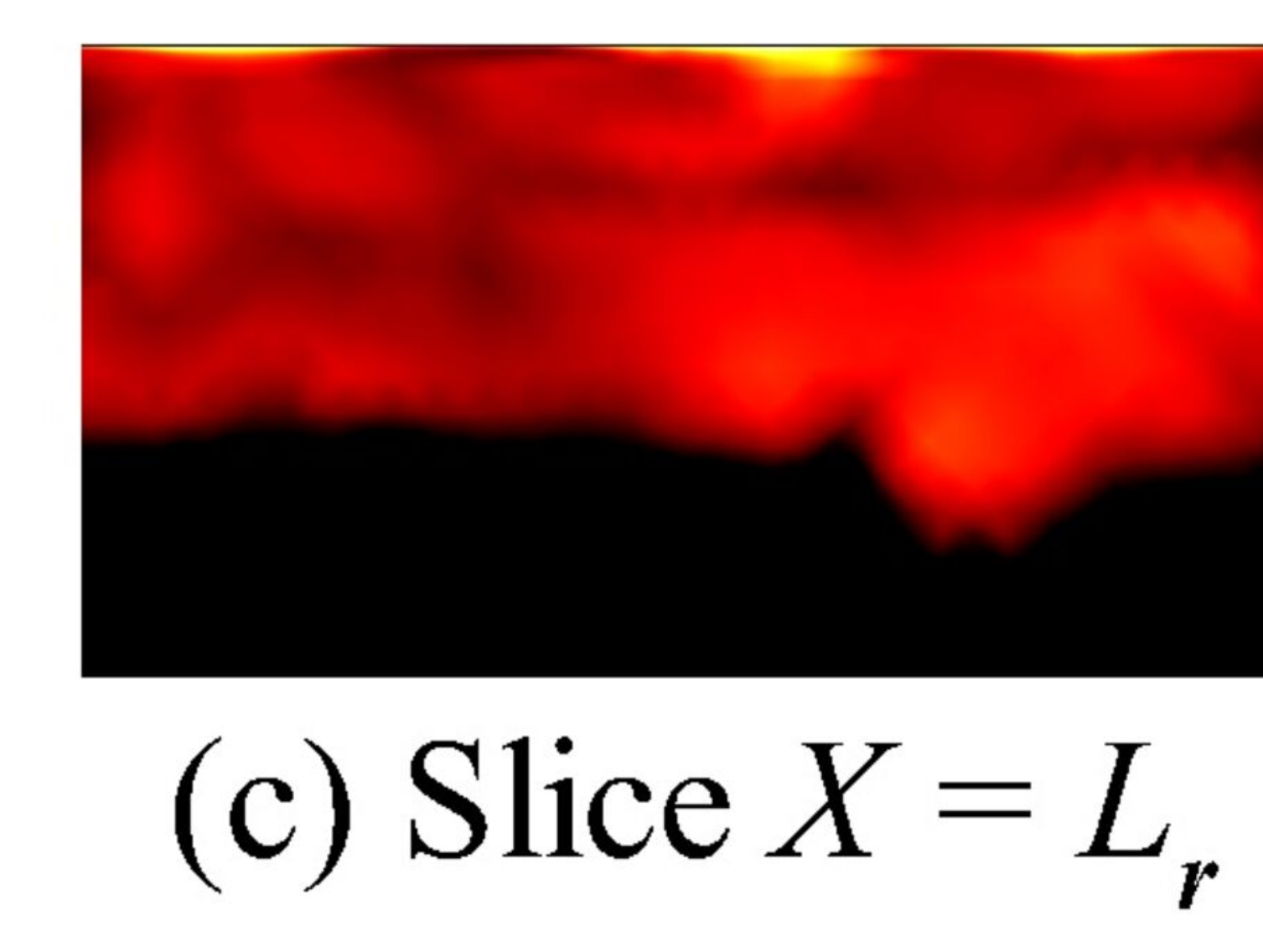

$\begin{array}{lllllllll}300 & 301 & 302 & 303 & 304 & 305 & 306 & 307 & 308\end{array}$ $T(K)$ 


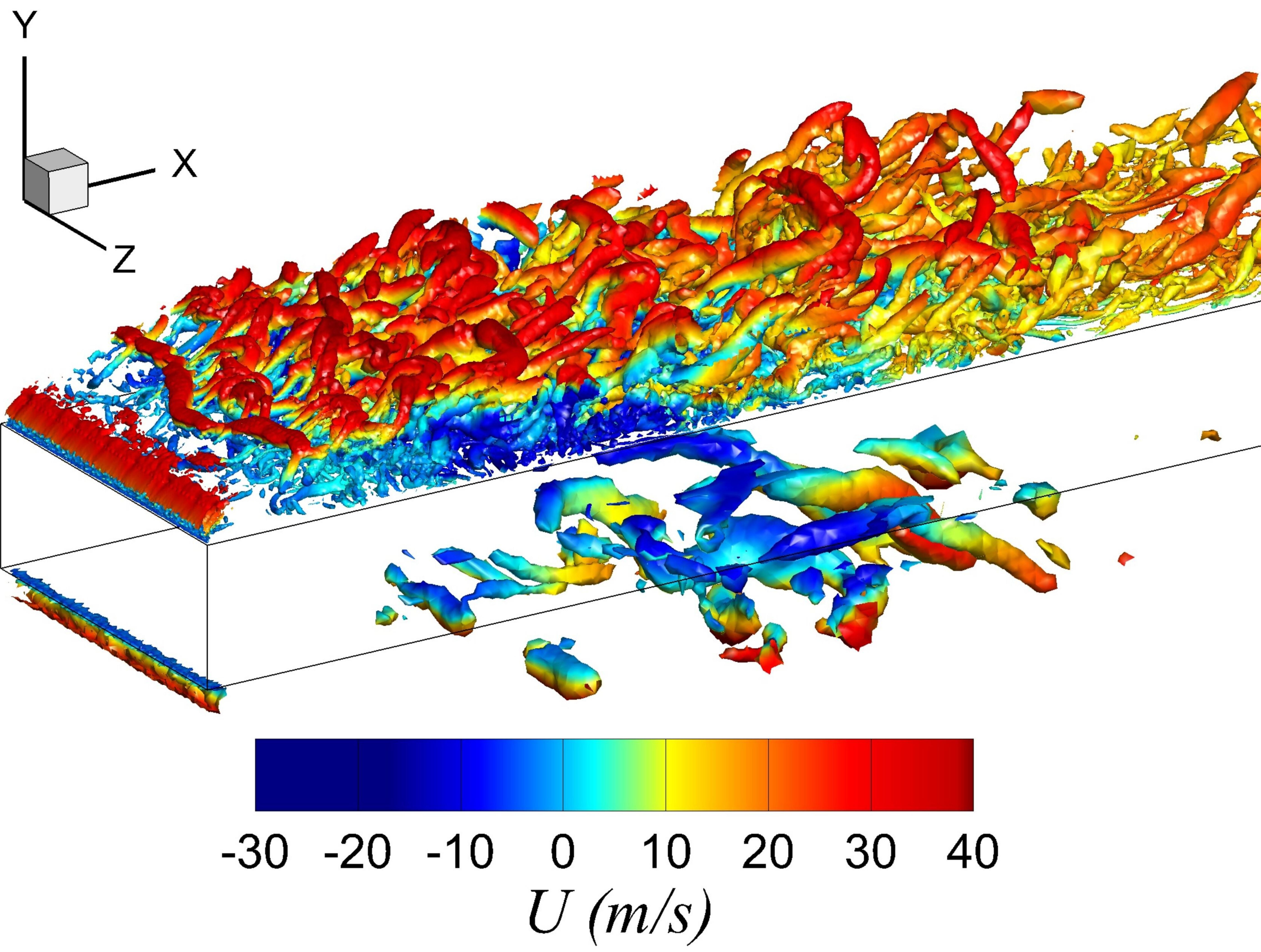




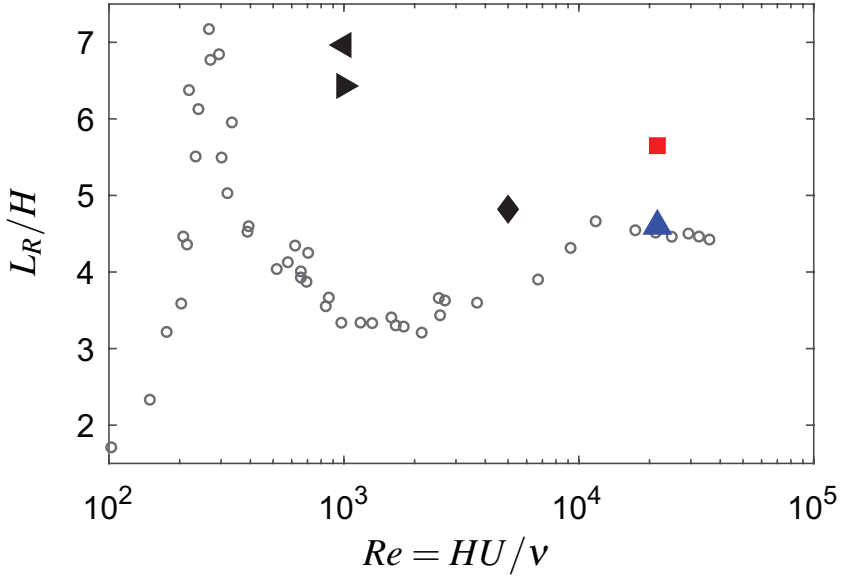




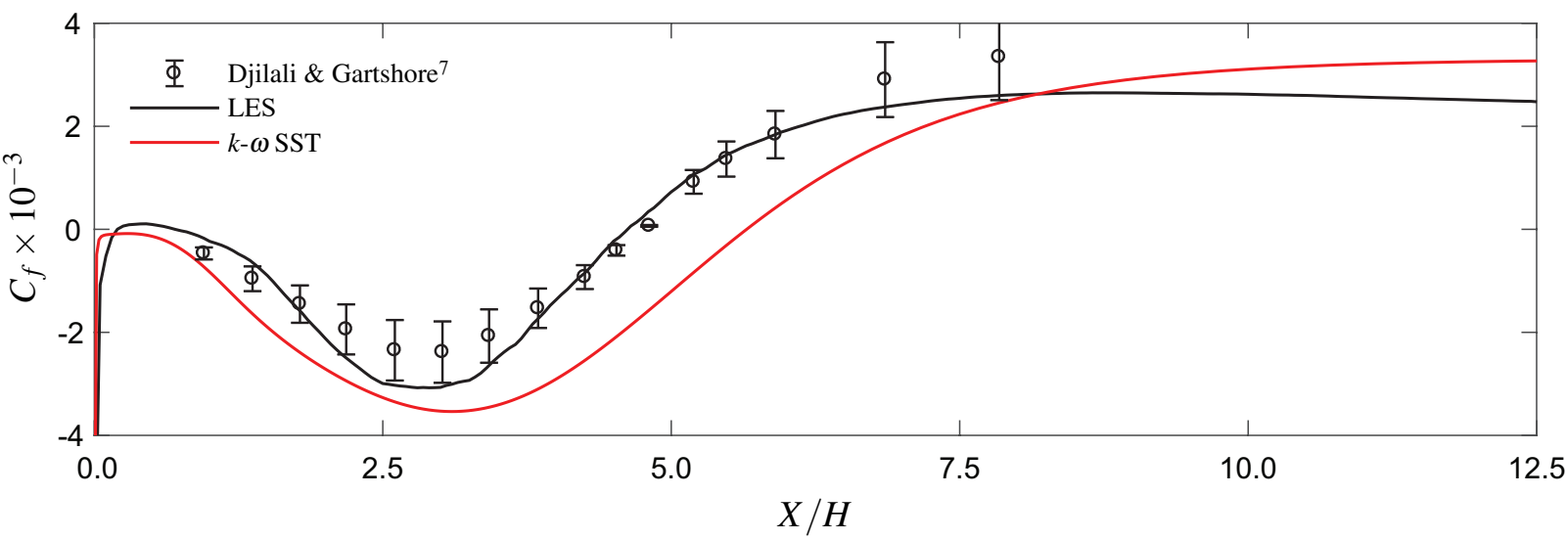




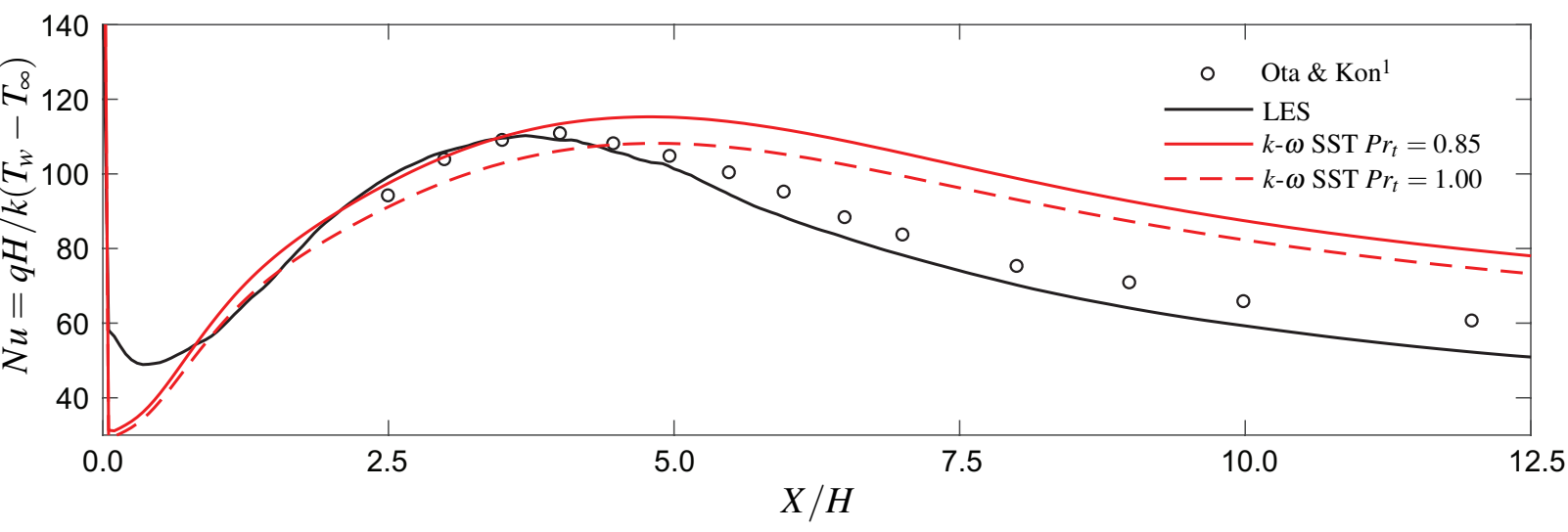




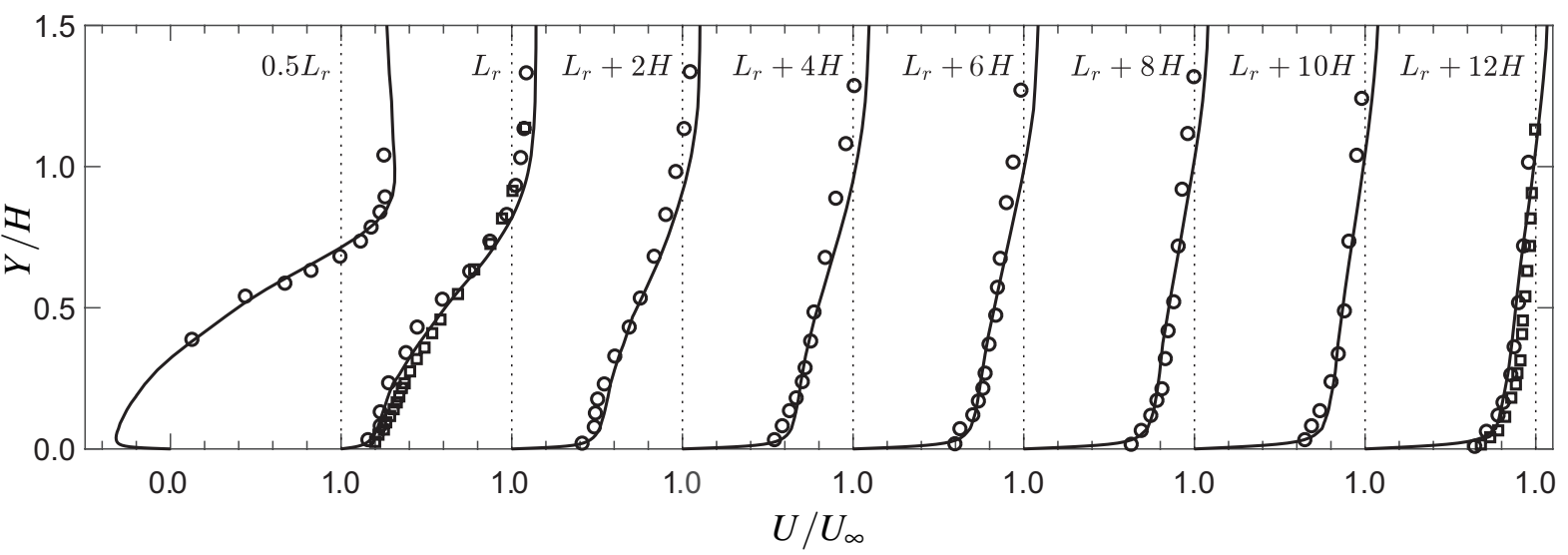




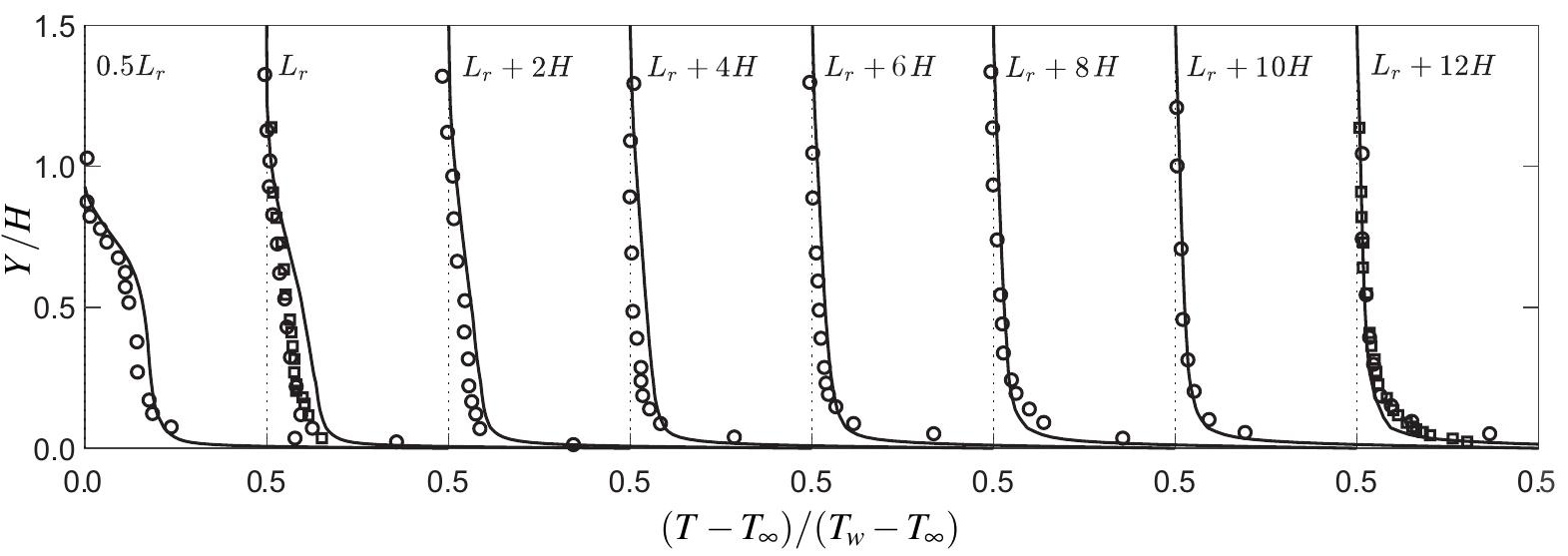




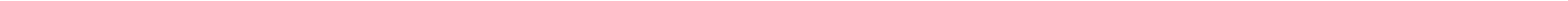


Three Comp.

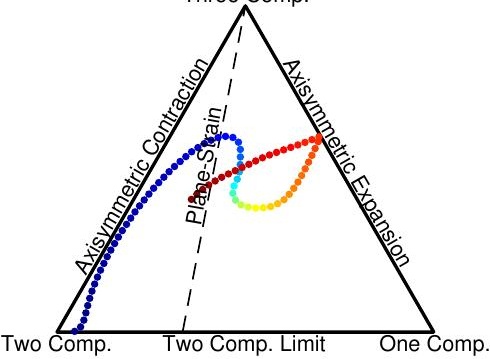


Three Comp.

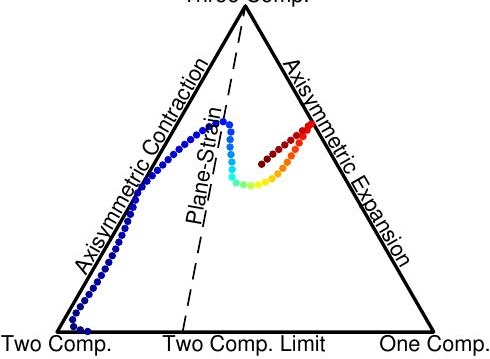


Three Comp.

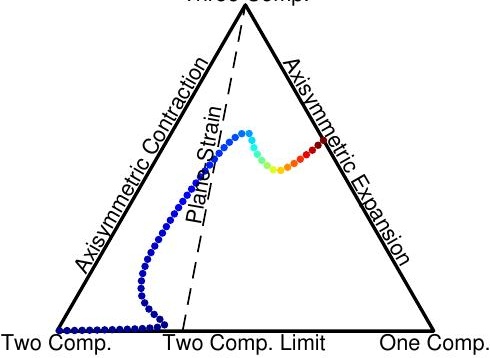


Three Comp.

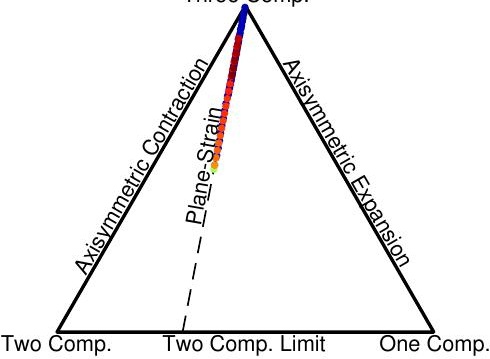




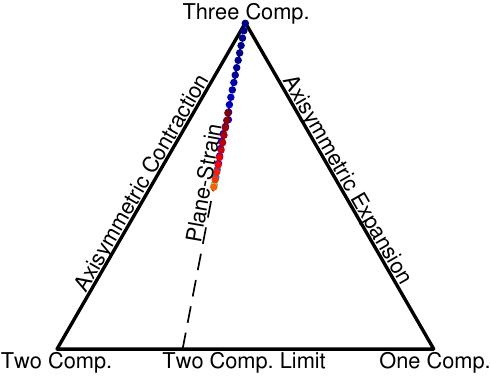


Three Comp.

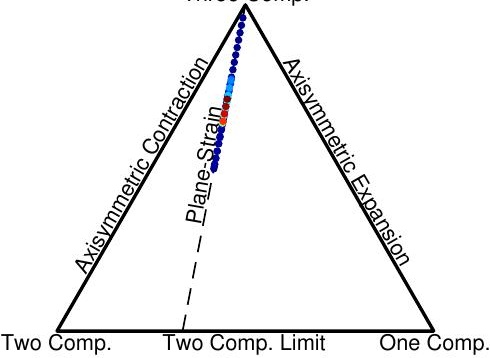


Three Comp.

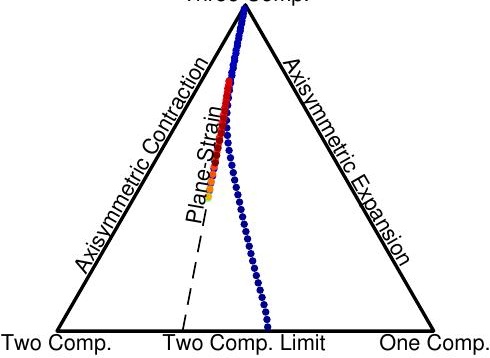


Three Comp.

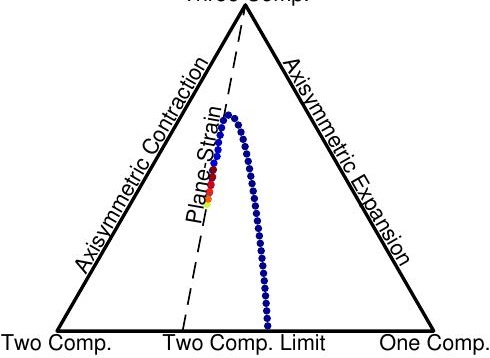


Three Comp.

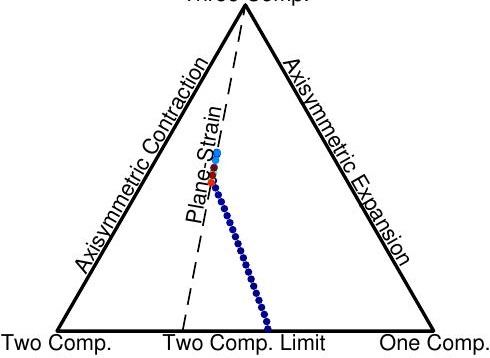




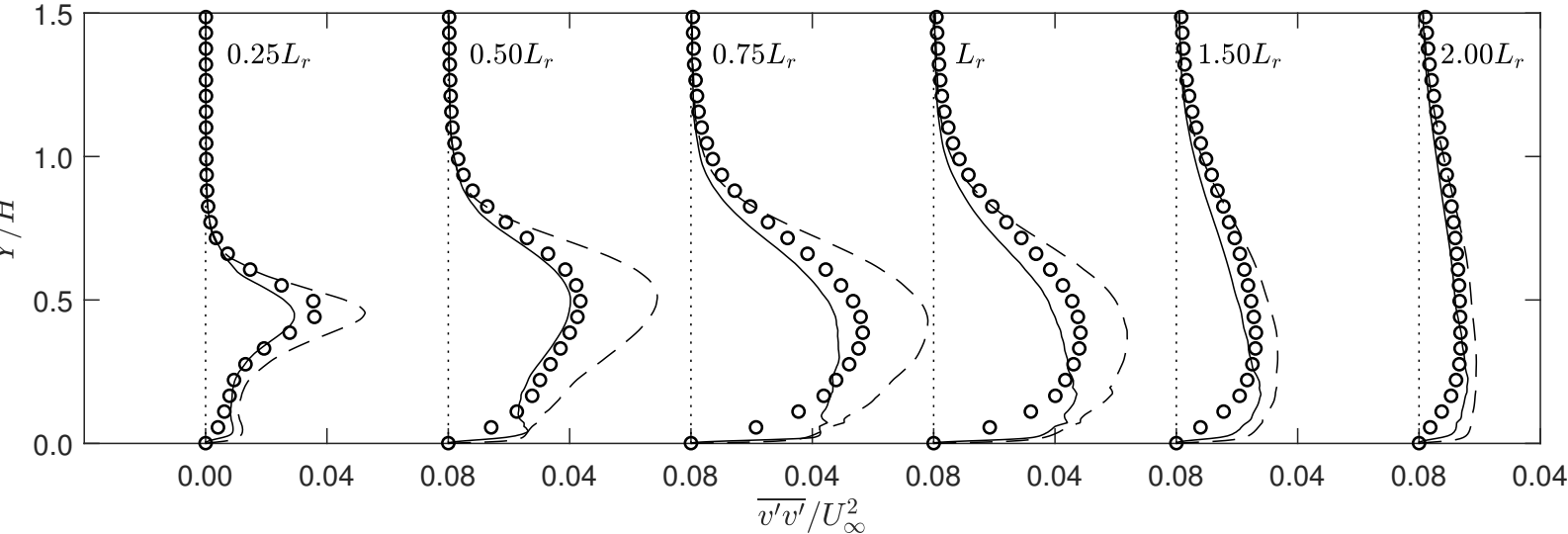




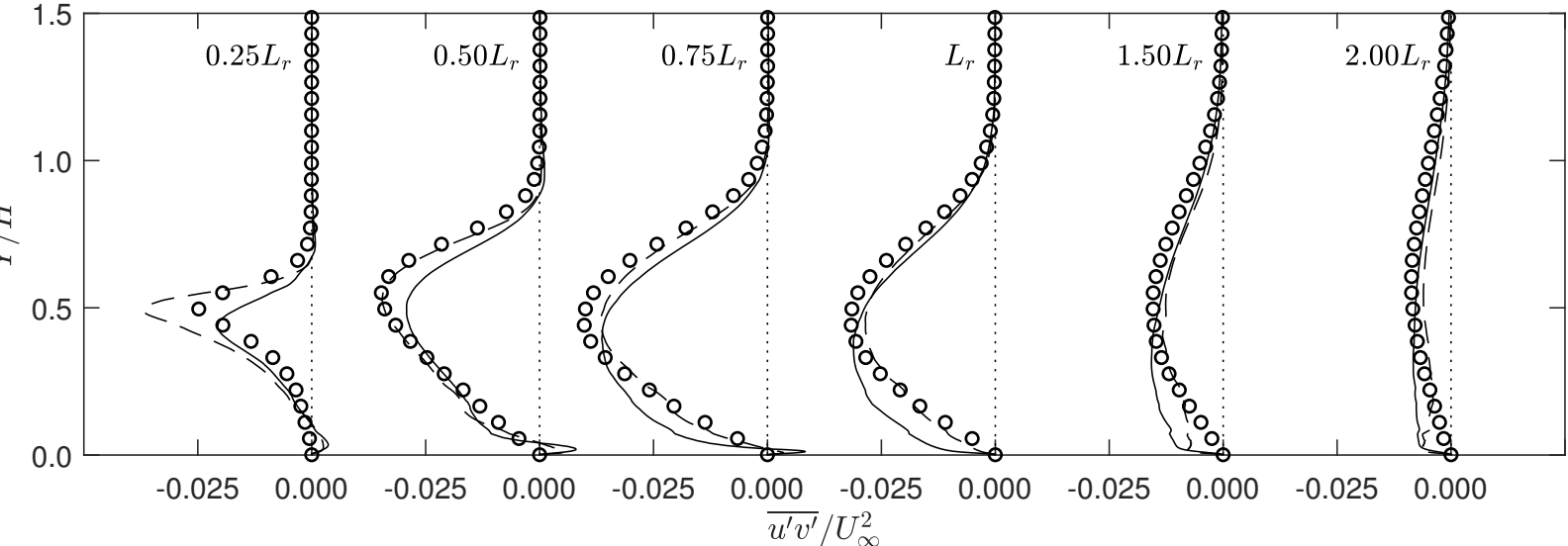



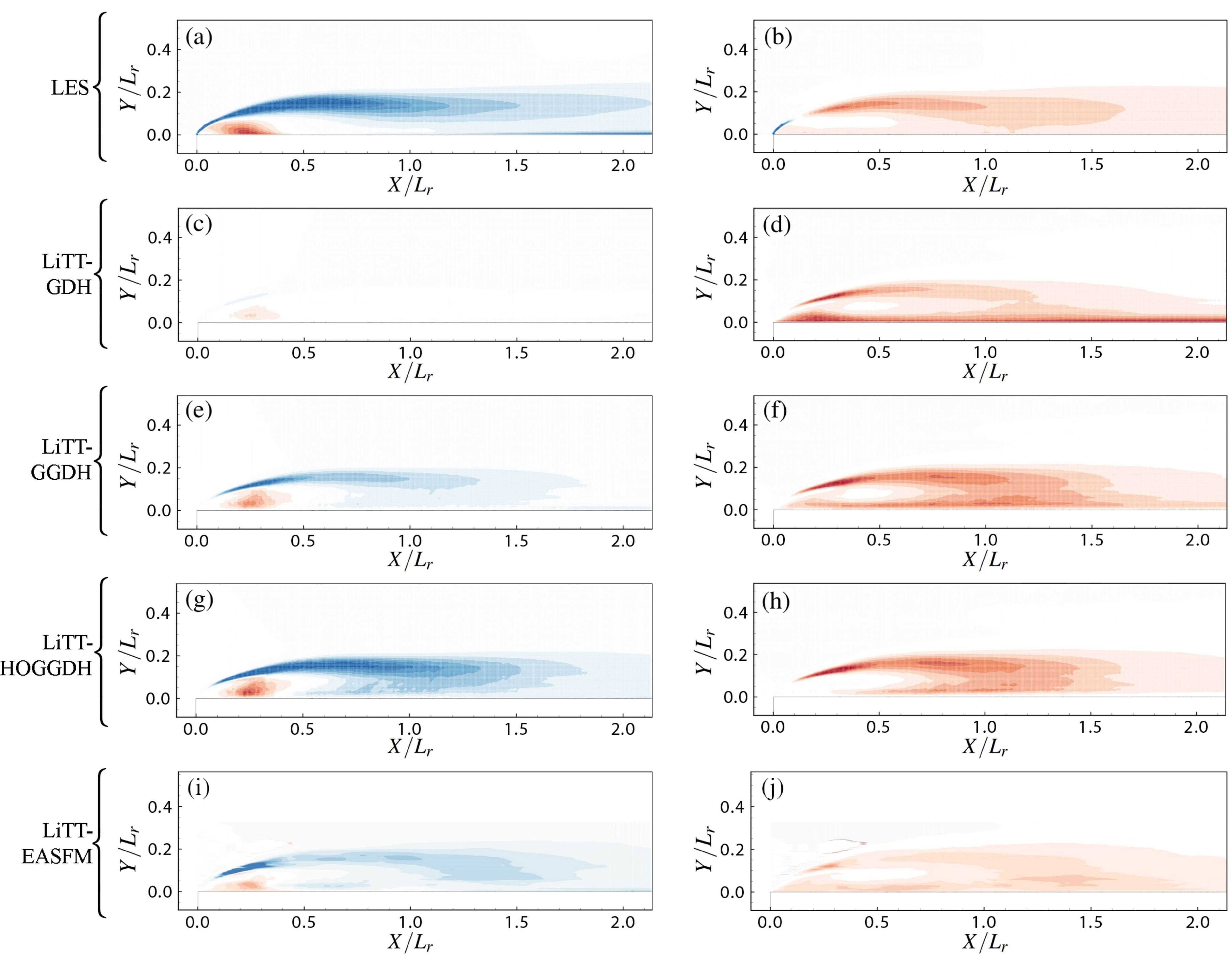


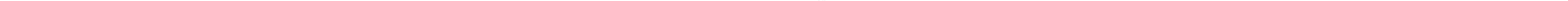




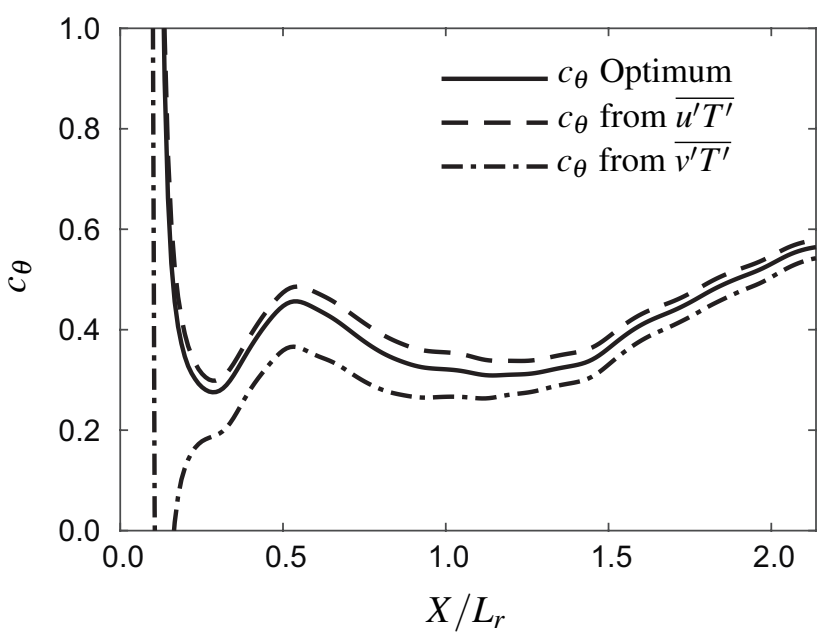




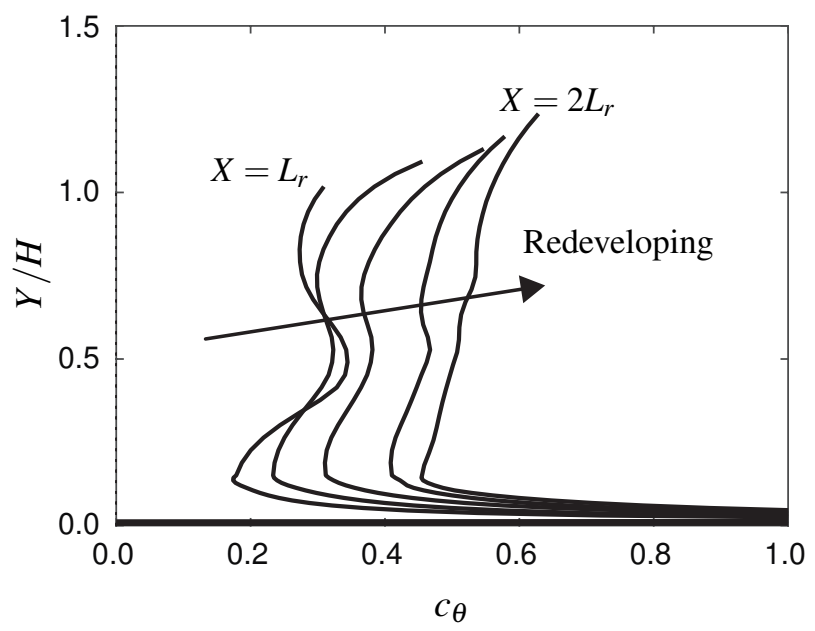




$$
\therefore=
$$




$$
\Gamma
$$





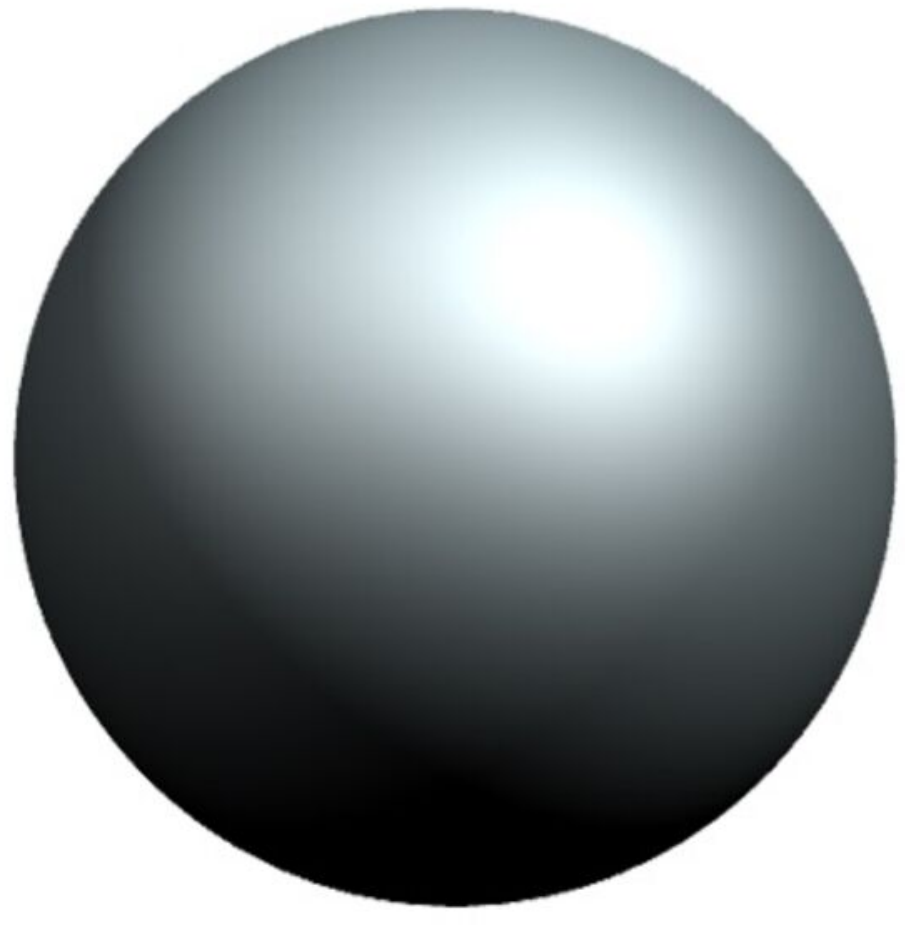


<smiles>[CH]</smiles> 


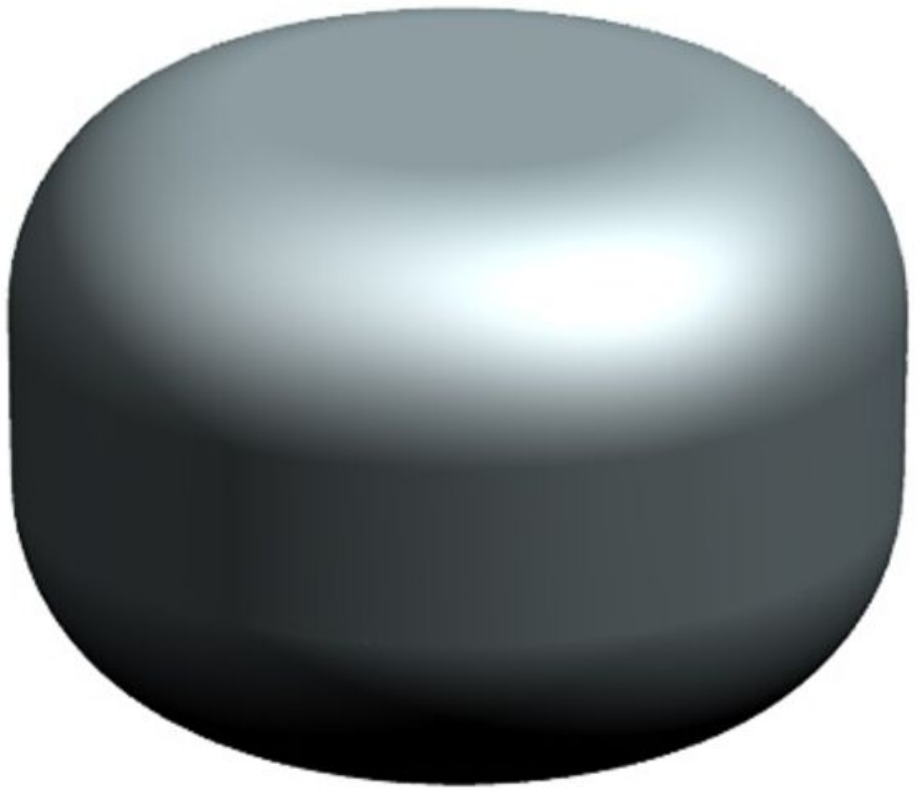




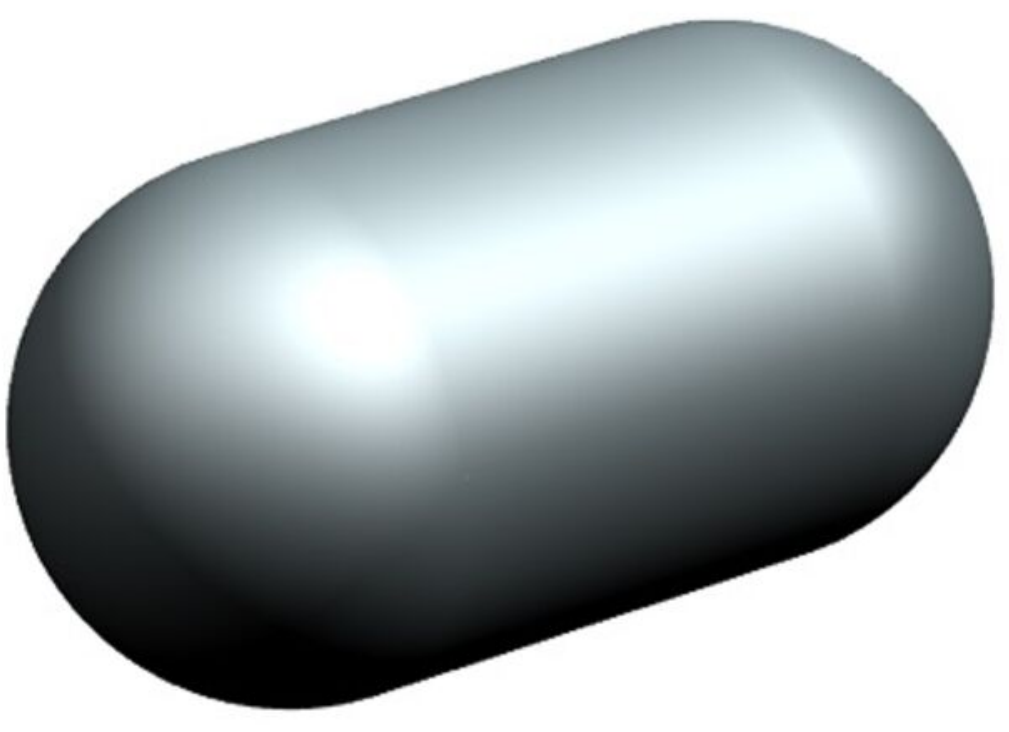




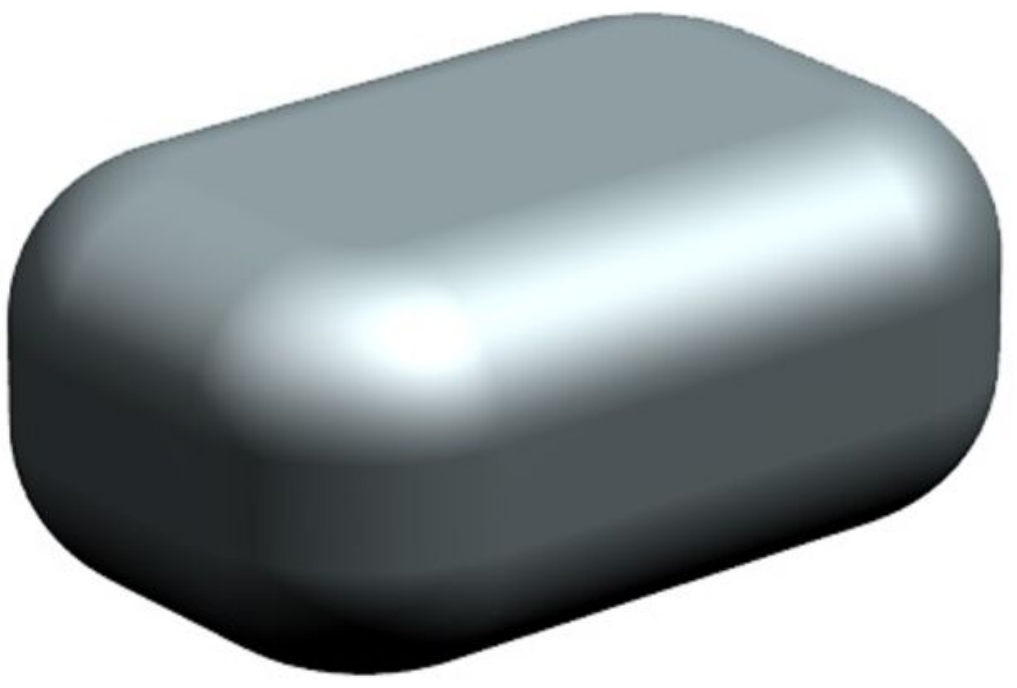

